\title{
Lessons Learned From Developing Reactor Pressure Vessel Steel Embrittlement Database
}

July 2010

Prepared by John Jy-An Wang

Oak Ridge National Laboratory

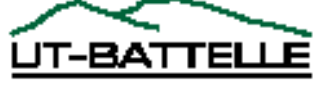

ORNL-27 (4-00) 


\section{DOCUMENT AVAILABILITY}

Reports produced after January 1, 1996, are generally available free via the U.S. Department of Energy (DOE) Information Bridge.

Web site http://www.osti.gov/bridge

Reports produced before January 1, 1996, may be purchased by members of the public from the following source.

National Technical Information Service

5285 Port Royal Road

Springfield, VA 22161

Telephone 703-605-6000 (1-800-553-6847)

TDD 703-487-4639

Fax 703-605-6900

E-mail info@ntis.fedworld.gov

Web site http://www.ntis.gov/support/ordernowabout.htm

Reports are available to DOE employees, DOE contractors, Energy Technology Data Exchange (ETDE) representatives, and International Nuclear Information System (INIS) representatives from the following source.

Office of Scientific and Technical Information

P.O. Box 62

Oak Ridge, TN 37831

Telephone 865-576-8401

Fax 865-576-5728

E-mail reports@adonis.osti.gov

Web site http://www.osti.gov/contact.html

This report was prepared as an account of work sponsored by an agency of the United States Government. Neither the United States government nor any agency thereof, nor any of their employees, makes any warranty, express or implied, or assumes any legal liability or responsibility for the accuracy, completeness, or usefulness of any information, apparatus, product, or process disclosed, or represents that its use would not infringe privately owned rights. Reference herein to any specific commercial product, process, or service by trade name, trademark, manufacturer, or otherwise, does not necessarily constitute or imply its endorsement, recommendation, or favoring by the United States Government or any agency thereof. The views and opinions of authors expressed herein do not necessarily state or reflect those of the United States Government or any agency thereof. 
Materials Science and Technology Division

\title{
LESSONS LEARNED FROM DEVELOPING REACTOR PRESSURE VESSEL STEEL EMBRITTLEMENT DATABASE
}

\author{
Jy-An John Wang \\ Materials Science and Technology Division \\ Oak Ridge National Laboratory
}

Date Published: July 2010

\author{
Prepared by \\ OAK RIDGE NATIONAL LABORATORY \\ P.O. Box 2008 \\ Oak Ridge, Tennessee 37831-6285 \\ managed by \\ UT-BATTELLE, LLC \\ for the \\ U.S. DEPARTMENT OF ENERGY \\ under contract DE-AC05-00OR22725
}




\section{Contents}

ABSTRACT …

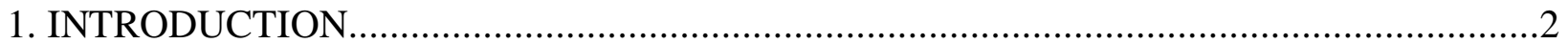

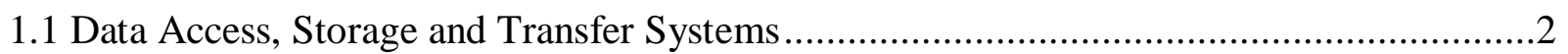

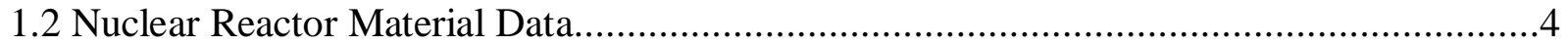

1.3 The Integrated Distributed Database Management System (IDDMS) ..................................4

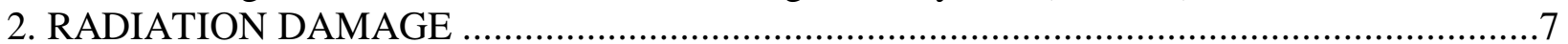

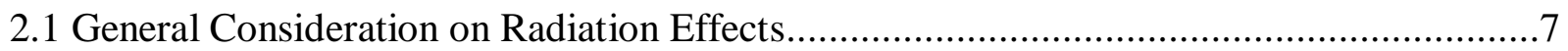

2.2 The Issue on Radiation Damage Unit...........................................................................

2.3 Metallic Material Behaviors in Response to Radiation ......................................................

2.4 Pressure Vessel Steels (Ferrite and Martensitic Steels)....................................................10

3. TYPICAL PROTOCOL USED FOR DEVELOPING DATA BASE FOR "RADIATION

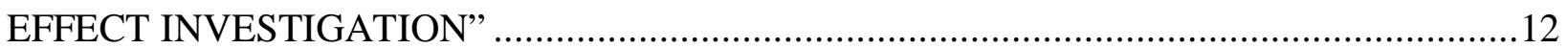

3.1 ORNL Embrittlement Database (EDB) Development ....................................................13

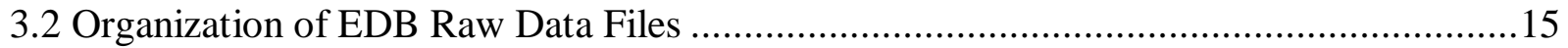

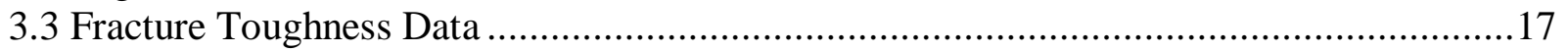

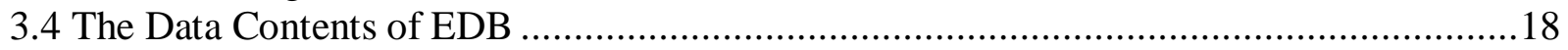

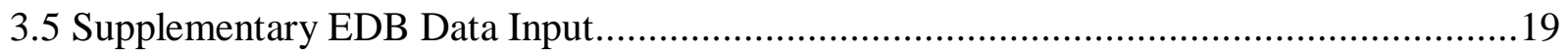

3.6 Dosimetry Database.................................................................................................19

3.7 Applications of EDB in Radiation Embrittlement of RPV Materials ...............................20

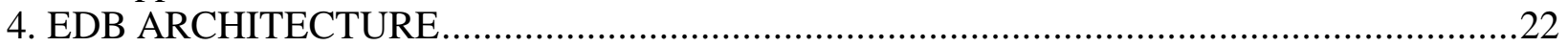

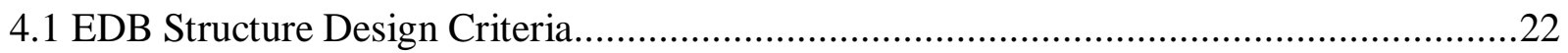

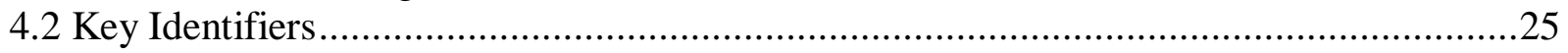

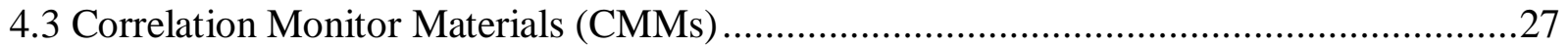

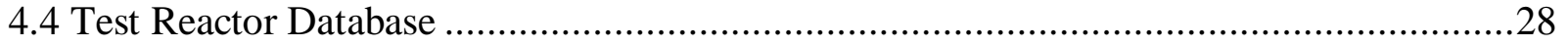

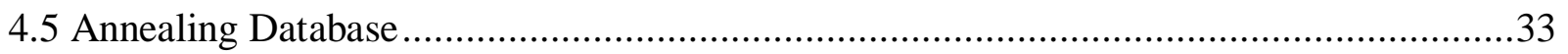

4.6 P-T Database and the Associated Software PT_LIM.....................................................34

5. IAEA INTERNATIONAL DATABASE ON REACTOR PRESSURE VESSEL

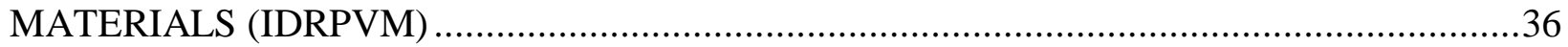

5.1 IAEA IDRPVM Aging Database Initiative ................................................................36

5.2 The Potential Benefits of IDRPVM Data Base ................................................................

5.3. Organization Mechanism for Developing IDRPVM Database..........................................37

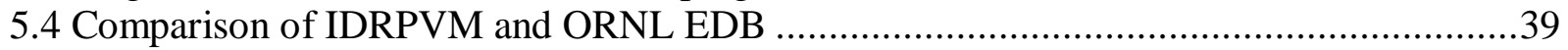

5.5 RPV Surveillance Data Presented at "First Meeting of Liaison Officers to the IAEA

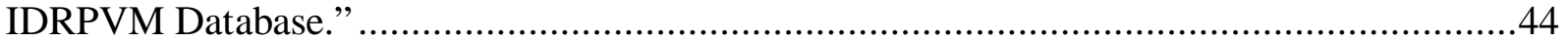

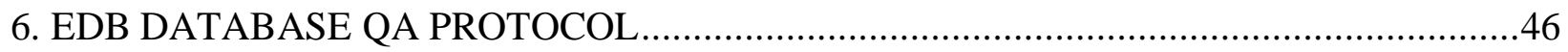

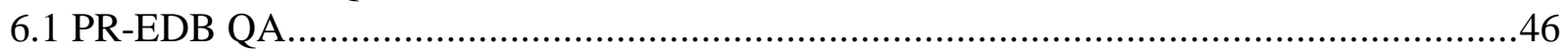

6.2 EDB Data Input Procedures.....................................................................................4

7. GENERAL CONSIDERATION OF MATERIAL DATABASE QA PROTOCOL.................49

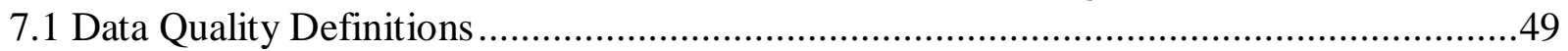

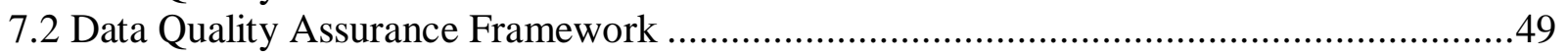

7.3 Material Database Quality Assurance Protocol .................................................................51

7.4 Other Consideration and Recommendation Regarding the QA Protocol............................52

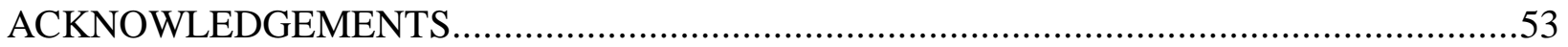

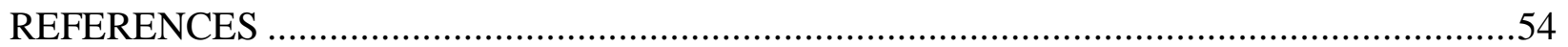




\section{Figure Content}

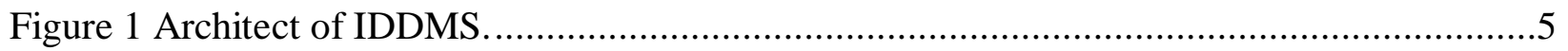

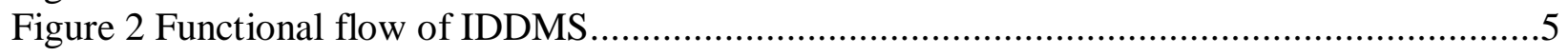

Figure 3 Central Server Functionality ...............................................................................6

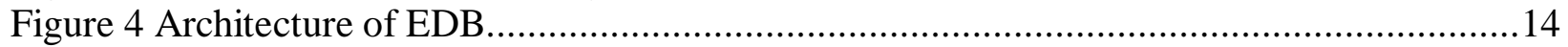

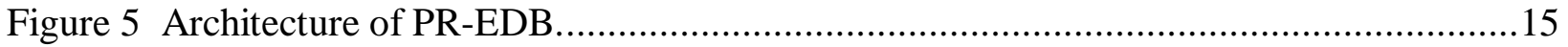

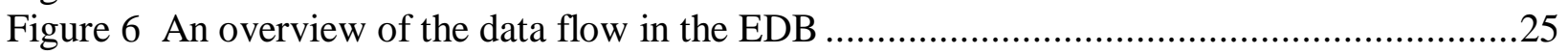

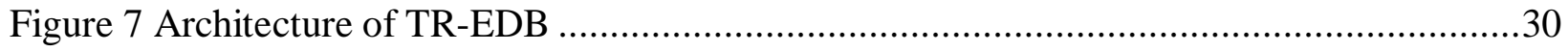

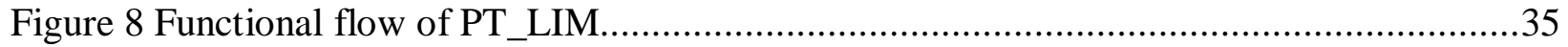

Figure 9 IAEA IDRPVM database structures ..........................................................................40

\section{Table Content}

Table 1 Units used in Embrittlement Database (EDB) files.........................................................24

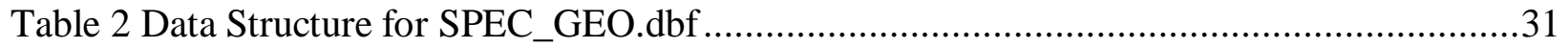

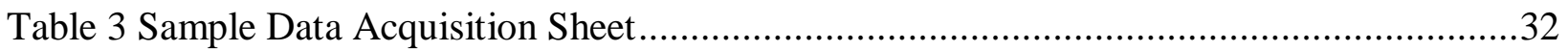

Table 4 Comparison of EDB and IDRPVM.....................................................................42

Table 5: Causes of error leading to insufficient data quality in a registry ..................................50

Table 6: A framework for data quality assurance in registries....................................................50 


\section{ABSTRACT}

Materials' behavior caused by neutron irradiation under fission and/or fusion environments cannot be understood without practical examination. Easily accessible material information system with large material database using effective computers is necessary for design of nuclear materials and analyses or simulations of the phenomena. The developed Embrittlement Data Base (EDB) at ORNL provides the comprehensive collection of data. The EDB contains power reactor pressure vessel surveillance data, material test reactor data, foreign reactor data (through bilateral agreements authorized by NRC), and the fracture toughness data. The lessons learned from building the EDB program and associated database management activity regarding "Material Database Design Methodology, Architecture and the Embedded QA Protocol" are described in this report. The development of IAEA International Database on Reactor Pressure Vessel Materials (IDRPVM) and the comparison of EDB database and IAEA IDRPVM database are provided in the report. The recommended database QA protocol and database infrastructure are also stated in the report. 


\section{INTRODUCTION}

Collection of specialized information have existed in academic research for many years, usually in the form of libraries attached to universities or in scientific and engineering societies. The National Bureau of Standards and Patent Office have long represented the federal governments in this field. In broad terms, the present day data bases consist of collections of documents, journal articles and their abstracts, and bibliographic compilations, as well as collections of numerical data, often in tabular form. Of interest here are the data bases of science and technology that contain information pertinent to nuclear/material engineering application. Once the information is entered on a magnetic medium in proper format and equipped with relevant keywords, or numerical code words, it can be loaded into a computerized retrieval system. The data files thus created can be queried either off-line by data base personnel, or on-line, by the end user. Data bases that provide an on-line searching capability help extend greatly the resources that are readily accessible to the nuclear and material engineers, provided the engineer is equipped with a suitable computer connected to an appropriate network.

\subsection{Data Access, Storage and Transfer Systems}

The Committee on Data for Science and Technology (CODATA) is an interdisciplinary committee organized under the International Council of Scientific Unions (ICSU). CODATA is concerned with all types of quantitative data resulting from experimental measurements or observations in the physical, biological, geological, and astronomical sciences. Particular emphasis is given to data management problems common to different scientific disciplines and to data used outside the field in which they were generated. The U.S. National Committee for CODATA (which is organized by the National Research Council) sponsored the first major interdisciplinary conference in December 1997 on the subject regarding the exchange of scientific and technical (S\&T) data among different computing environments, and across diverse scientific and engineering disciplines.

This CODATA initiative covered many important issues relating to data exchange. By data exchange is meant several things: the transfer of large amounts of data from one set of software to another software; extracting small amounts of data from one or more data sources for specific use; and the creation of a linked or integrated data system with multiple data sources. Data exchange has two major components: the stream of bits and bytes that actually represent the data items and fields, and the contextual meaning of individual data items and fields. S\&T disciplines and applications have begun addressing data exchange issues, but progress has been slow and difficult for a variety of reasons. Scientists are often not accustomed to formal standards. Discipline experts, even though they may be quite knowledgeable in computation and database management, frequently lack expertise in information modeling and exchange standards. Metadata are not well defined, complicating the application of data across diverse scientific areas. As a result, interdisciplinary data exchange has been difficult to promote and rarely implemented. For example, the geographic information, many applications need such information: to locate physically the sources of samples, to describe the range of a phenomenon, or to specify the location of an event, among others. Today many geographic information 
systems serve diverse communities of users, and several efforts to develop standards for exchanging data among these systems have been proposed. Yet progress to develop such standards in other areas has been slow. Other types of scientific data, such as biological nomenclature, chemical and engineering material identification and temporal data, suffer the same problem. Many uses for these data exist outside the scientific disciplines that generate them, yet accepted methods for exchanging these data remain elusive.

From U.S. National Committee for CODATA documented case studies, "The Challenge of Combining Diverse Environmental Data," in which data interfacing, defined in that report as the coordination, combination or integration of data for the purpose of modeling, correlation, pattern analysis, hypotheses testing, and field investigation at various scales, was necessary to achieve full value of research investment. Data interfacing is founded upon the standards and protocols agreed to by different scientific disciplines to exchange data. Particular emphasis must be put on the role of metadata in this data exchange.

The need to develop standardized representations is well understood. Standards have the advantage of providing a published and stable representation; and standards have a change process that is based on consensus. Proprietary exchange formats can and do change at the discretion of companies and research institutes that developed them. Further, manufacturing is increasingly global. To be more effective, the standards for exchanging product information must gain worldwide acceptance. A single international standard, ISO 10303 - Product data representation and exchange, has been designed to meet this industrial need. This standard is more commonly referred to as STEP, Standard for Exchange or Product model data. The standardized product data representations all use an object description language called EXPRESS to define the content of the data to be communicated. The actual communications interfaces, a message/file format and an application-programming interface, are independent of any particular implementation technology.

The introduction of harmonious cooperation among heterogeneous hardware and software systems carries the potential for great increases in productivity and improvement in application processing. The use of these heterogeneous systems requires an integrated system that would allow the development of global applications requiring communication and cooperation among the pre-existing systems. An InterBase System was proposed to overcome this heterogeneity barrier. It integrates pre-existing systems over a distributed, autonomous, and heterogeneous environment to support global applications while retaining local autonomy.

To achieve integration (integratable systems) requires at least compatibility of data representation, communication paradigms, and system architectures. Advances have been made in each of these areas, such as product data exchange (STEP - ISO 10303), communication protocols (TCP/IP, OSI) and architectures (CIM Framework, CORBA). Although it is the information that must be shared between these systems, it is the representation and language that provides the mechanism to allow the sharing to take place. One area of data representation which has received relatively little attention is process data. A generic process specification language (PSL) program for describing process, for the need to share process information in the manufacturing environment, is currently funded by NIST. 


\subsection{Nuclear Reactor Material Data}

While in the past the industrial and national laboratories carried the bulk of design and development work on nuclear reactors, the task of operating and managing nuclear installations fell, for the most part, to the private utility industry. The industry experienced the need for supporting organizations to orchestrate activities affecting all companies in that field, primarily in research, and in the collection and evaluation of operating data. In response to that need, three organizations were created by the users, and financially supported by their contributions. The principal organizations created by the utility and reactor vendor industry are: the Atomic Industrial Forum, the Electric Power Research Institutive, and the Institute for Nuclear Power Operations. Thus, well designed infrastructure is needed to provide efficient information bridge among the academic, research institution, regulatory agency, and nuclear industry.

Materials' behavior caused by neutron irradiation under fission and/or fusion environments can be little understood without practical examination. An easily accessible material information system with large material database using effective computers is necessary for design of nuclear materials and analyses or simulations of the phenomena. The author has recommended developing an Integrated Distributed Database Management System (IDDMS) to approach the above criteria.

The IDDMS is constructed on the computer network which connects engineering workstations in the separate organizations. A relational database management system is used. A distributed material database is based on the hardware with specially designed common data structure. Data storage has been carried out continuously in each organization. The equipment of useful userinterface system, such as retrieval, data entry, and process supporting and image data handling systems, have also been constructed to make it friendly for the user. The supporting analytical software is also under development from different research groups.

This integrated system will greatly enhance the information flow and speed up the development of advanced materials, and can bring about technical breakthrough in many areas of interest. Especially, it can be considered that these will bring out remarkable improvements in the performance of nuclear plants and components. The success of IDDMS will be based on the cooperation of nuclear industry, research institutes, and regulatory agency. The main research activity fields of these constituents may be different from each other to some extent; thus, with broaden the point of view on materials research.

\subsection{The Integrated Distributed Database Management System (IDDMS)}

The IDDMS is proposed and will include three major aspects stated as below:

- Generate Common Databases and Application Programs

- Build Networks, Intellectual Interfaces, to carry out Data Sharing and Data Exchange

- Develop Long Term Archive Methodology 
The general tasks that can be evolved from the above mentioned aspects are demonstrated in the following flow chart. The tasks above the dash line are related to the central server, which will be managed and operated at ORNL. The architect and function flow of IDDMS are illustrated in Fig. 1 and Fig.2, respectively.

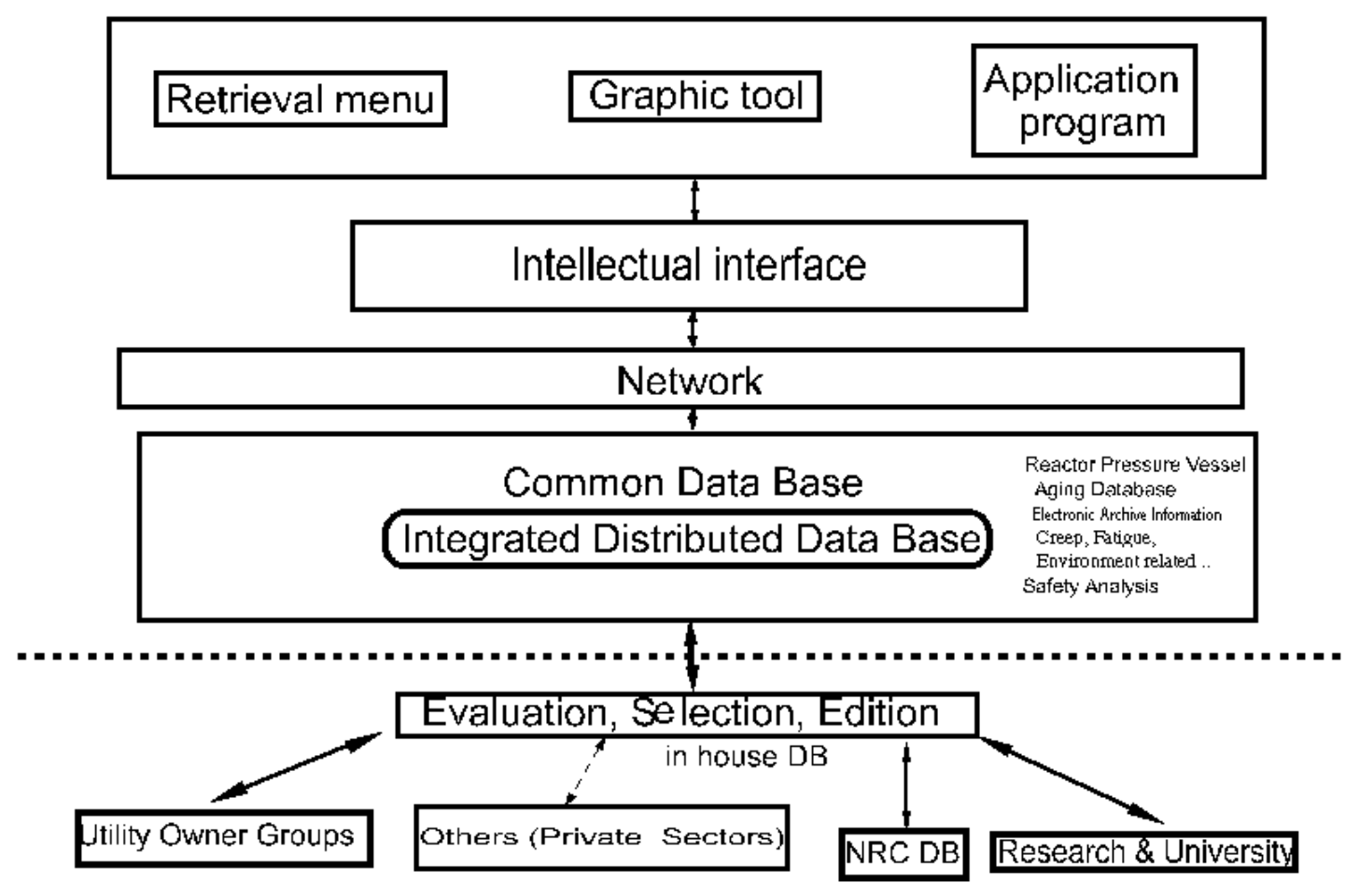

Figure 1 Architect of IDDMS.

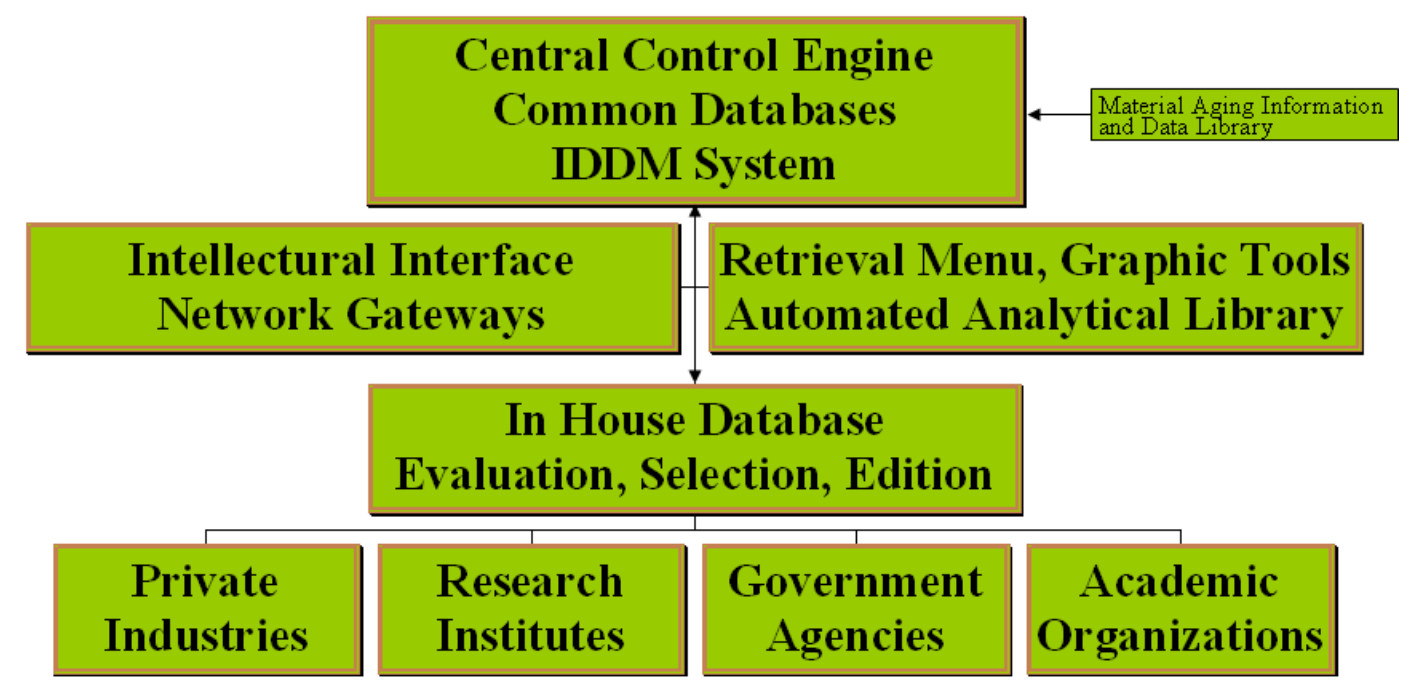

Figure 2 Functional flow of IDDMS 
The central server that resides at ORNL, will provide the linking mechanism among the technical community, regulatory or funding agencies and nuclear power industry, as illustrated below.

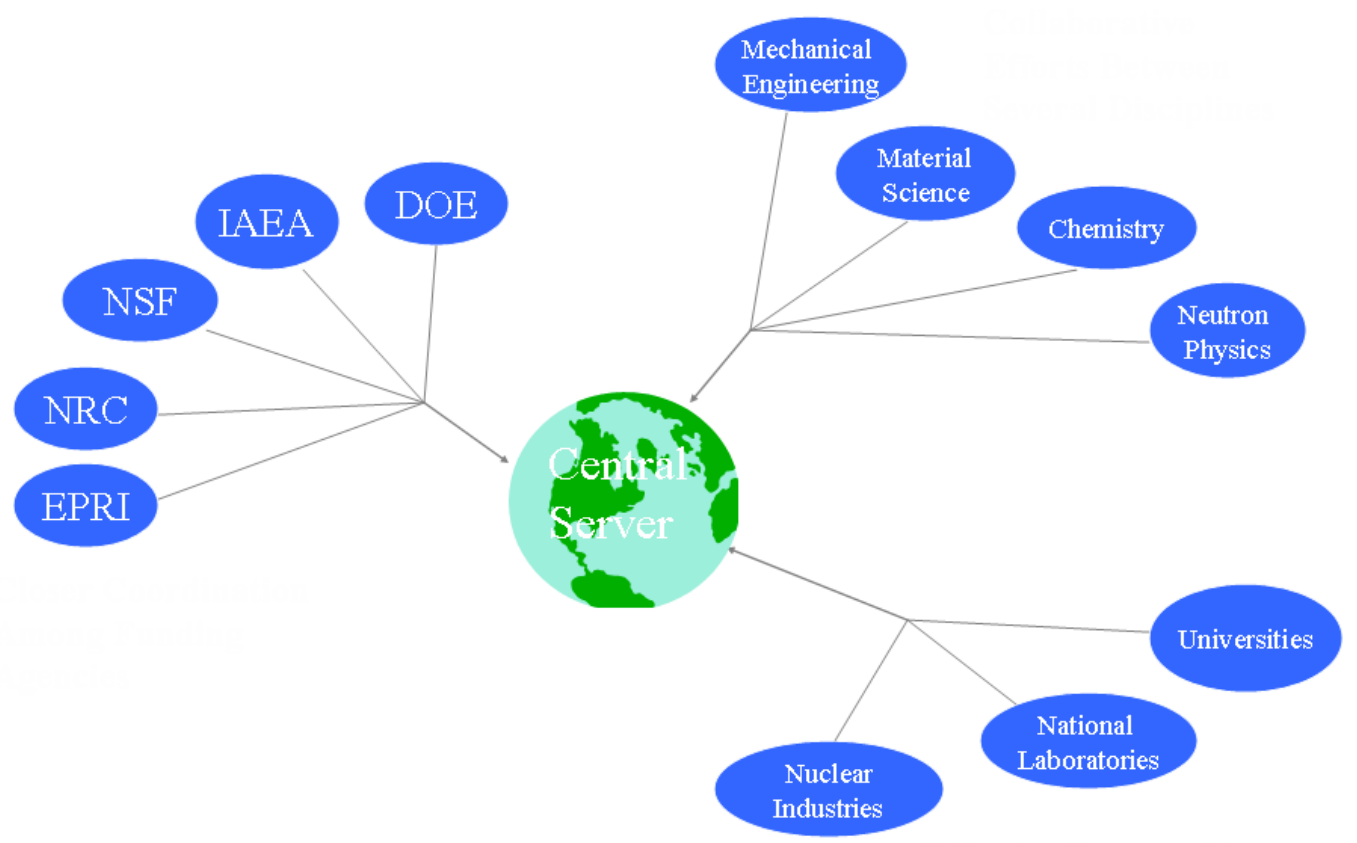

Figure 3 Central Server Functionality

The general contents for each aspect of IDDMS are described as below.

- Generate Common Databases and Application Programs

Develop unified format and data structures for the common databases

$>$ Utilize visual object-oriented program to develop upgrade version of EDB

$>$ Generate automated programs and macros to create an analytical program library to carry out the following tasks:

- Updating of analyses whenever new data or new assumptions for the analysis arise,

- Follow-up studies with refined models, optimizing procedures, exploring consequence in what-if scenarios,

- Documentation and opportunity for critical review by NRC or other affected parties.

- Build Networks, Intellectual Interfaces, to carry out Data Sharing and Data Exchange

$>$ Construct intellectual interface for easy data access and data sharing

$>$ Build networks among NRC, Utilities, Research Institutes, Universities

- Develop Long Term Archive Methodology

$>$ Design strategy to store or restore old data tapes and diskettes.

$>$ Select standard format to convert the data source into common databases.

$>$ Convert hard copy documents into electric data files. 


\section{RADIATION DAMAGE}

\subsection{General Consideration on Radiation Effects}

By understanding the underlying mechanisms of the radiation damage, the associated neutron energy and flux evaluation, and the inherited metallic material characteristics will further strengthen database designers' capability to construct a well-designed material database for the specific applications and provide a basis to develop a meaningful and easily implemented QA protocol. In general, the QA protocol is embedded within a well-designed database automatically, i.e. the methodology of the QA protocol is directly translated into the architect of the designed database structure.

Effects of radiation on solids have been studied for over 150 years. Of much more interest to the present discussion, however, are the intense radiation fields in reactors. That the success of reactor technology would depend critically on the choice of high-temperature material with satisfactory neutronic properties was pointed out by Fermi in 1946. Wigner is generally credited with the first suggestion that fluxes of energetic neutrons and fission fragments in neutron chain reactors would displace significant numbers of atoms and thus alter physical and mechanical properties.

Swelling was discovered in 1967 as a result of electron microscopic observation of a stainless steel that had been irradiated in the Dounreay Fast Reactor in Scotland. Cavities were found ranging in size from the resolution of the electron microscope of few nanometers up to more than $150 \mathrm{~nm}$. Macroscopic swelling was confirmed by immersion density measurements. Irradiation creep was known somewhat earlier. The work was on uranium metal, which exhibited irradiation creep under neutron irradiation. Irradiation creep strains are generally smaller than swelling strains. Irradiation embrittlement of austenitic stainless steel and Inconel was first reported in 1959, when it was noted that lower ductility was observed in tensile and stress-rupture tests after irradiation.

The basic changes produced by irradiation on solids are:

Swelling

$>$ Irradiation Creep

$>$ Ductility Loss

$>$ Microstructure and Microcomposition

$>$ Mechanism: Radiation-induced processes in metals and alloys can be thought of in two broad divisions.

- Damage production, or radiation damage, concerns the initial events in the processes embracing the displacement of atoms.

- Radiation effect encompasses the kinetics of processes resulting from the atomic defects so produced and the concomitant evolution of microscopic structure composition and macroscopic properties these induce. 
Generally the atom-displacing reactions that occur in fission and fusion reactor structural materials are induced by neutrons. These include $(n, \gamma),(n, n),(n, p),(n, \alpha)$, and $(n, 2 n)$ reactions., where $\gamma$ denotes gamma ray, $n$ ' denotes a neutron of a different energy, $\mathrm{p}$ denotes proton and $\alpha$ denotes the helium nucleus. Following the displacement events the energy of the disturbed region is degraded ultimately to thermal energies. The point defects and small-defect clusters relax and diffuse. Typically during diffusion most vacancies and interstitials are annihilated by mutual recombination. Some avoid recombination, however, and these are responsible for radiation effects. These defects are either absorbed at preexisting sinks such as the grain boundary or dislocations, or aggregate with like defects to produce extended defect clusters. Most of defects absorbed at sinks ultimately recombine there. However, some are left to accumulate. When the defect clusters become large enough they are often observed as cavities or dislocation loops.

At the same time, by diffusion, the point defects can induce preferential flows of alloying elements toward or away from sinks. Demixing of the alloy results in local regions being highly enriched or depleted in alloying constituents. Often the phase transformation occurs when the local solubility limit for precipitation is exceeded.

\subsection{The Issue on Radiation Damage Unit}

The primary source of neutron radiation damage in metals is the displacement of atoms from their normal lattice sites. Hence, an appropriate damage exposure index is the number of times, on the average, that an atom has been displaced during irradiation. Dpa, the average number of times of displaced atoms per atom of the material, is in wide used as an exposure index and as a correlation parameter.

The absolute accuracy of the dpa calculation is strongly affected by the fluence spectrum, the reaction cross section used in calculating $\sigma_{\mathrm{d}}(\mathrm{E})$, the Lindhart model for the partition of energy between atoms and electrons, and the conversion of deposited energy to displacements. The relative accuracy of dpa calculations for different environments depends on energy dependence of $\sigma_{\mathrm{d}}(\mathrm{E})$ and on the relative accuracy of fluence-spectrum determination.

The dpa is a measure of the potential to create point defects, therefore it is only the first logical step in the determination of the production of the lattice defects that are responsible for changes in the mechanical and physical properties of materials. It is now believed that hardening and embrittlement due to irradiation does not depend on the initial displacement per atom in the metallic lattice, but on the "residual defects" after one accounts for possible recombination of vacancies and interstitials in the metallic lattice. In particular, neutron, and some charged particle irradiations produce high energy pka which, in turn, produces high localized concentrations of displaced atoms and vacant sites. The high defect concentration in these displacement cascades enhances the probability that a displaced atom will recombine with its own or some other vacant lattice site and thus anneal out potential damage. Hence, the number of residual defects produced, rather than the total number of displacements, is an improved measure of damaging exposure. 
This residual defect depends on several factors: 1) The temperature of the material, 2) the rate at which the fluence is accumulated, and 3) the energy of the neutrons causing the displacements. Higher temperatures, higher flux levels, and higher energy neutrons all enhance recombination effects.

Generally speaking, dpa is a good first approximation for the correlation of radiation embrittlement, for the flux that consists mainly of fast neutrons, the differences in pka spectra from various neutron spectra are not expected to strongly affect the point defect availability factor. However, additional modification needs to be considered for those spectrums having high thermal/fast neutron ratio, or the displacement damage energy deposited by thermal neutrons is within an order of magnitude of that deposited by fast neutrons.

\subsection{Metallic Material Behaviors in Response to Radiation}

Some of the general characteristics of metallic materials used in power reactor environments are described below.

\section{$\underline{\text { Ferrite and Martensitic Steels }}$}

The smallest radiation-induced increases in Nil-Ductility-Transition temperature (NDT) take place in steel that is low in interstitial elements (carbon, oxygen, nitrogen), has a small grain size, and possesses a quenched and tempered microstructure. Post-irradiation annealing at $400^{\circ} \mathrm{C}$ to $450^{\circ} \mathrm{C}$ results in significant restoration of the baseline material.

\section{$\underline{\text { Austenitic Stainless Steel }}$}

The maximum hardening of austenitic stainless steels by irradiation is produced at temperature of about $300^{\circ} \mathrm{C}$. The changes in tensile properties can be removed by annealing at 500 to $600^{\circ} \mathrm{C}$. A drastic reduction in the ductility of irradiated austenitic stainless steels takes place at elevated temperatures, which is presently attributed to helium bubbles formed at grain boundaries, and cannot be recovered by annealing at temperature up to $1350^{\circ} \mathrm{C}$. Helium is present as a result of the thermal-neutron ${ }^{10} \mathrm{~B}(\mathrm{n}, \alpha)^{7} \mathrm{Li}$ reaction as well as form $(\mathrm{n}, \alpha)$ reaction between fast neutrons, and iron, nickel, and other alloy constituents. The magnitude of the ductility loss is increased by large grain size and low strain rates.

Nickel Base Alloys

Radiation affects the mechanical properties of nickel-base alloys in about the same way as it does for austenitic stainless steels.

\section{$\underline{\text { Refractory Metals }}$}

For niobium, molybdenum, rhenium, tungsten, and vanadium alloys, the magnitude of radiation effects on the mechanical properties of these materials was found to be very sensitive to the 
interstitial content of the materials. Increased interstitial content resulted in larger radiationinduced increase in ductile-to-brittle transition temperature. The required annealing temperature for removal of the radiation-induced changes in mechanical properties generally increases with increasing melting temperature of the alloy. The refractory metals do not exhibit the radiationembrittlement at elevated temperatures that is common to austenitic stainless steels and nickel alloys, and the stress-rupture properties are not significantly altered by irradiation.

\subsection{Pressure Vessel Steels (Ferrite and Martensitic Steels)}

The nuclear industry has used ferritic steels as structural materials in reactor application for many years. Mostly, these steels have been used for pressure vessel construction. Since the ferritic steels have a body-centered crystal structure, their fracture mode changes from ductile to brittle with decreasing temperature. Other characteristics are,

Composition: $\mathrm{P}, \mathrm{Si}, \mathrm{Mo},(\mathrm{C}>0.2 \%)$

Grain Size: $\quad$ steels with smaller grain size have been found to have lower NDT temperatures.

Cold Work: cold working raised the NDT of steels, and aging following cold work results in further increasing in NDT, cold working by cyclic fatiguing results in considerable increase in NDT with the increase being largest when the temperature is in the 400 to $580^{\circ} \mathrm{F}$ range.

Heat treatment: A quenched and tempered structure has a considerably lower NDT than an annealed or normalized structure.

Welding: Welding generally results in higher NDT because the heat-affected zone resulting from welding is similar to that of annealed metal.

General Consideration

Material variability needs to be taken into account for a specific HEAT, since the reported chemistry may not necessary represent the specimen used for testing.

Higher uncertainty exists in irradiation temperature.

$100 \%$ recovery of upper-shelf energy (USE) of Charpy-V Notch (Cv) trend curve does not mean a full recovery of all the irradiation induced defects.

Below $650^{\circ} \mathrm{F}$ annealing of fast neutron damage mechanism is essential negligible.

Different material heat treatment may play an important role on $\Delta$ NDT.

FCC lattice has less resistivity Frenkel Pairs

$\mathrm{Cu}<\mathrm{Al}<\mathrm{Ni}<\mathrm{Fe}<\mathrm{Cr}<\mathrm{Zr}$

fcc bcc hcp 
Pre-irradiation heat treatment will affect the $\mathrm{Cv}$ curve during the irradiation environment. For example longer heat treatment can reduce NDT shift \& $\triangle$ USE (drop), in some case the USE even increased. There is evidence that other mechanisms are responsible for the reduction of the upper shelf than for the transition temperature shift.

Ni \& Cu have a synergetic effect on delta TT30 but may not on $\triangle$ USE. Also at high fluence, Ni influence on delta TT30 is unfavorable, probably favorable at low fluence.

At low $\mathrm{Cu} \& \mathrm{Ni}$ a completed saturation curve was observed.

The mechanism of annealing is a combination of the reversible \& non-reversible processes. Therefore, the true recovery ought to consider the re-irradiation effect for those unstable defects that recover right after the irradiation and before the initiation of stable defects.

The copper precipitation model has been extremely successful in explaining many aspects of irradiation embrittlement. However, there is increasing evidence that there are other important mechanisms contributing to the embrittlement of RPV steel, including $\mathrm{Ni}, \mathrm{P}, \mathrm{Mn}, \mathrm{Mg}$, voidmicrovoid.

Irradiation temperature effect: defect region average diameter, $255^{\circ} \mathrm{C}$ (9 micron) and $350^{\circ} \mathrm{C}(17$ micron).

The rate effect may not only state that more defect per dose for low flux irradiation but also imply that the more stable and inhomogeneous distribution of defects will be generated in low flux situation (longer irradiation) than that in the high flux irradiation environment. Therefore, the test reactor (TR) accelerated irradiation may generate more homogeneous defects (evenly distributed point defects), which will have more recovery through the annealing process, due to high recombination rate, the annealing recovery by TR may well overestimate the annealing recovery effect for PWRs.

Since the different damage mechanisms are competing with each other, it is not easy to define the influence of a single element or parameter.

Base and weld category are mainly reflected from their transition temperature curve.

Both Nickel and Manganese are helpful in lowering the initial transition temperature and the question arises as to whether a material more that is highly alloyed with these elements would tend to give a less harmful product when irradiated. The material with a further increased austenite content has better unirradiated properties, but this advantage is lost on irradiation. Cracking in the ferrite seems to govern behavior.

Strain aging: Nitrogen may be responsible for the irradiation of steel at $130-230^{\circ} \mathrm{C}$, or irradiation below $100^{\circ} \mathrm{C}$ followed by post irradiation heating at $200^{\circ} \mathrm{C}$ results in additional embrittlement. The nitrogen may result from decomposition of aluminum nitride in thermal spike. 


\section{TYPICAL PROTOCOL USED FOR DEVELOPING DATA BASE FOR "RADIATION EFFECT INVESTIGATION"}

In Phase I, collected data need to be streamlined and grouped into three major categories, namely, material history, service environment, and experimental results. An identification system (key identifiers) needs to be designed and to be implemented into user-friendly software. Each record will have the reference source identified, such as a report or published paper. The graphical reference will be scanned and stored in Tiff or PDF format. The detailed components of each category are listed below:

(1) Material History; which includes the following subcategories,

a) Material specification

b) Vendor \& Supplier

c) Manufacture \& Production information

(2) Service Environment; which includes the following subcategories,

a) Reactor name, location, and operation organization.

b) Neutron and gamma evaluation data, and short description of the method used.

c) Service environment, including temperature, irradiation, etc.

d) Stress environment, such as thermal, electrical, chemical, or mechanical stresses.

(3) Experimental test results; which will include the measurement of the material property in particular service environment by specific experimental procedures, and includes the following subcategories

a) Test results.

b) Testing procedure, specimen types.

c) System information, such as machine type, capacity and capability, etc.

d) Operating Engineering, and testing Laboratory.

In phase II, the development of the user-friendly software will be initiated, which will cover the development of the front end, utilities for data manipulation, plot and final report.

(1) Front end; which includes icons, and selection manuals for a user to select a specific task.

(2) Data manipulation; which will includes file manipulation features to allow the user to link and to process data files, and to create view on the screen.

(3) Generate final report or plot to a file or to a printer.

The above methodology was implemented in developing Embrittlement Data Base (EDB) for reactor pressure vessel steels. 


\subsection{ORNL Embrittlement Database (EDB) Development}

The aging and degradation of light-water-reactor (LWR) pressure vessels is of particular concern because of their relevance to plant safety and the magnitude of the expected irradiation embrittlement. The radiation embrittlement of RPV materials depends on many different factors such as flux, fluence, neutron energy spectrum, irradiation temperature, pre-irradiation material history and its chemical compositions. These factors must be considered to reliably predict the pressure vessel embrittlement and to ensure the safe operation of the reactor. Based on embrittlement predictions, decisions must be made concerning operating parameters, lowleakage-fuel management, possible life extension, and the need for annealing of the pressure vessel. Large amounts of data obtained from surveillance capsules and test reactor experiments are needed, comprising many different materials and different irradiation conditions, to develop generally applicable damage prediction models that can be used for industry standards and regulatory guides. The Embrittlement Data Base (EDB) provides this comprehensive collection of data resulting from the merging of the Power Reactor Embrittlement Data Base (PR-EDB) and the Test Reactor Embrittlement Data Base (TR-EDB). The fracture toughness data were also integrated into EDB.

The scope and purpose of this program, which has been sponsored by the Nuclear Regulatory Commission (NRC) since 1987, can be summarized as follows:

1. Compile and verify a comprehensive collection of data from power reactor surveillance programs, and test reactor irradiation experiments of pressure vessel materials from U.S. and foreign laboratories.

2. Provide software support for the use of the data base by furnishing programs and maintaining compatibility with commercially available software.

3. Facilitate the exploration and verification of embrittlement prediction models.

4. Facilitate the exploration and verification of the effects of annealing for pressure vessel life extension.

5. Interact with standards organizations to provide the technical bases for voluntary consensus standards that can be used in regulatory guides, Standard Review Plans, and codes.

To achieve these goals, the design of the data base architecture was made after many discussions and planning meetings with prospective users, materials scientists and members of research staff. The EDB is designed for use with any personal computer using the DOS base system. (Recently, the beta Windows version EDB was also completed) The data format that was chosen for EDB is dBASE; this format was initially introduced by Ashton-Tate and is now the virtual standard for relational data bases. This format allows queries and data processing not only with the current dBASE software but also with any of the now numerous "Xbase" developer tools, such as Foxpro or MS-ACCESS. The dBase files can also be imported into most other data base, spreadsheet, and word processing programs that run in the DOS or WINDOWS environment. The more recent versions of these programs contain extensive facilities for generating reports including statistic, curve fitting, and graphic programs. For often-performed tasks, customized EDB software based on Clipper or Visual Studio and FORTRAN that were written originally for PR-EDB, can be utilized with EDB. This EDB-Utility is menu-driven and self-explanatory requiring no special training. 
The data collections of EDB originated from the Material Properties Council (MPC) data base, which contains both power and test reactor data. From this collection an unpublished version of the EDB was constructed and has been augmented with more recently reported data. All data are traceable to the source of references, including page numbers. The architecture of the data base, shown in Fig. 4, is characteristic of a relational data base that makes it relatively simple to maintain and perform quality control.

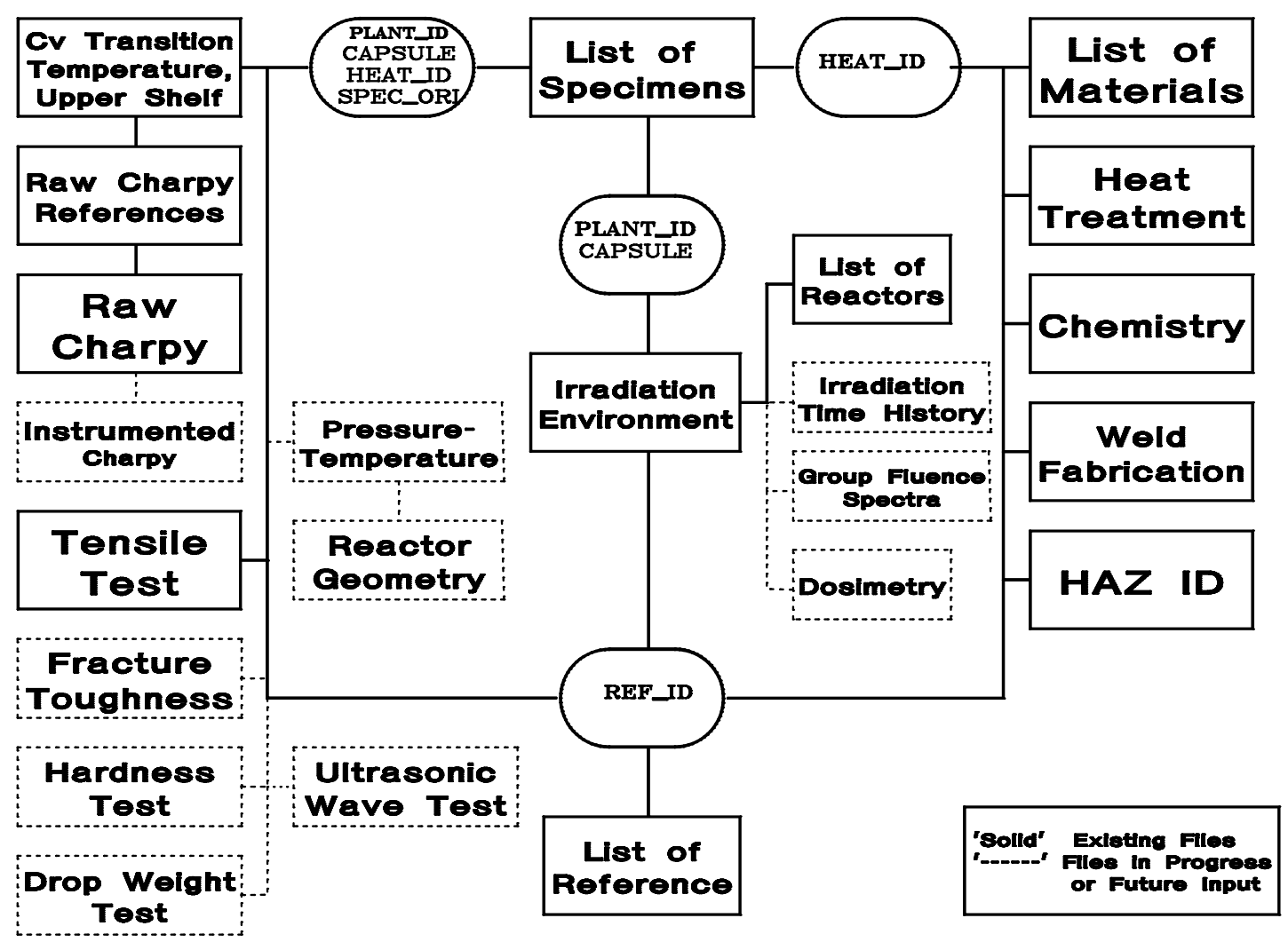

Figure 4 Architecture of EDB.

A restricted version containing only power reactor surveillance data - the PR-EDB - was assembled under NRC sponsorship to be used primarily for regulatory purposes. Most of the surveillance data listed in PR-EDB have been verified by the reactor vendors responsible for the insertion of the test material into surveillance capsules, and any changes and corrections have been documented in special files for future reference. Version 1 of PR-EDB was released to the public in July 1991. An updated Version 2 was released on January 1994. The architecture of PR-EDB is illustrated in Fig. 5. In the meantime the assembly and review of the MPC and other test reactor results has continued, tracing them to the original reports and adding more data. Significant additions came from NRC-sponsored investigations at Materials Engineering Associates, Inc. (MEA) and Oak Ridge National Laboratory (ORNL), the International Atomic Energy Agency (IAEA)-sponsored program, and a variety of other irradiation experiments at laboratories in France, Germany, Japan, and the United Kingdom. The verification of these test reactor data, TR-EDB, is difficult and cannot be as thorough and comprehensive as in the PR- 
EDB, mainly because of the way the results were reported. An additional problem is that laboratories and researchers responsible for the published data are often no longer available or cannot be funded for the considerable work required for outside reviews. All data have been checked internally for correctness and consistency, and all unresolved problems are reported in the "NOTES" field. Every effort is being made to resolve discrepancies by contacting the original investigators. Version 1 of TR-EDB was released in January 1994.

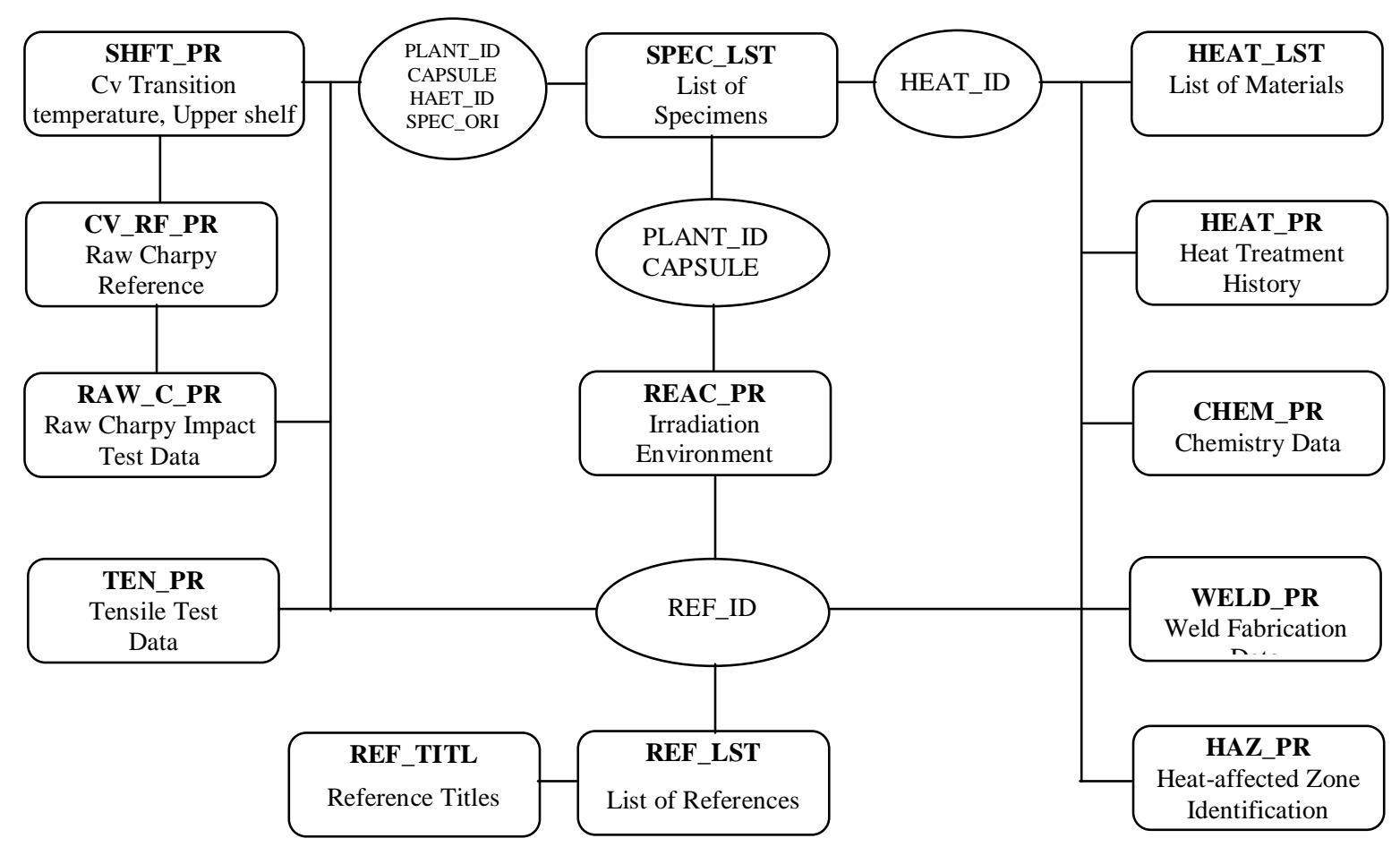

Figure 5 Architecture of PR-EDB.

\subsection{Organization of EDB Raw Data Files}

The organization of the current version of PR-EDB is shown in Fig. 5. At the top center of the PR-EDB architecture is the file SPEC_LST.dbf containing a complete list of test specimen sets that are used in the commercial surveillance programs. The specimen sets can be uniquely identified by SPEC_TYPE and four key identifiers; PLANT_ID, CAPSULE, HEAT_ID, and SPEC_ORI. Specimen sets for testing the baseline properties of unirradiated materials are characterized by leaving the CAPSULE field blank.

Radiation embrittlement data are distributed over three major categories in PR-EDB: preirradiation material history, irradiation environment, and mechanical test results. The detailed architecture of the EDB data is also illustrated in Fig.5 and descriptions of each major category of data follow: 
- The first category (left-hand side of Fig.5) includes results of material mechanical property tests. Charpy, both individual tests and results of curve fittings and tensile are currently available (SHFT_PR.dbf, RAW_C_PR.dbf, and CV_RF_PR.dbf for Charpy data; TEN_PR.dbf for tensile). Each record in these files is uniquely characterized by the combination of PLANT_ID, CAPSULE, HEAT_ID, and SPEC_ORI.

- The second category (middle of Fig. 5) contains data describing the reactor and radiation environment for each surveillance capsule. The file REAC_PR.dbf contains a detailed description of the fluence, irradiation temperature and irradiation time for each irradiation capsule. This file links with the others via the key identifiers PLANT_ID, and CAPSULE. This linkage will not be needed in most cases because fluence and irradiation temperature are also given in the data files, such as SHFT_PR.dbf and TEN_PR.dbf. Note that the fluence values given in REAC_PR.dbf apply only for the capsule as a whole (i.e., average or capsule center), which is not necessarily the same as for the individual specimen or specimen group listed in the test data files. However, many details such as values for fluence $(\mathrm{E}>0.1 \mathrm{MeV})$ or dpa are listed, when available, in REAC_PR.dbf. The file REAC_LST.dbf (see Table 6 for a list of irradiation facilities used in the experiments). The key identifier field in this file is PLANT_ID. The development team is considering the addition of more detailed files containing the irradiation history, the group fluence spectra, and dosimetry data to allow for fluence determination by independent investigators and more detailed investigation on the rate effect, spectrum effect, and residual defects to the embrittlement prediction models.

- The third category (right-hand side of Fig. 5) contains information about the chemical composition and fabrication of materials used in RPV surveillance programs. HEAT_LST.dbf lists all HEAT_IDs with reported codes, and CHEM.dbf, HEAT.dbf, WELD.dbf, and HAZ.dbf list the actual chemical composition and fabrication data. HEAT_ID links these files to the rest of the PR-EDB files.

Most records in the PR-EDB files include a reference in the field REF_ID that includes one or more page numbers which permits verification of the data sources and points to additional information. Exceptions are given in the file REAC_LST.dbf, in which information comes from many different sources. References are not listed in RAW_C_PR.dbf because the associated file CV_RF_PR.dbf has the necessary references. A detailed list of all references with complete title, authors, and date of publication is given in the REF_TITL.dbf. REF_ID and is the link to the other files.

PR-EDB files with the suffix _LST are somewhat different from the other data files in that they provide a sort of directory of the other files and their relations to key identifiers. SPEC_LST.dbf is a directory of capsules and baseline specimen sets. REAC_LST.dbf is a directory of power reactors. Finally, HEAT_LST.dbf is a directory of the materials contained in the PR-EDB files.

The effort and coordination with the NRC research staff, EPRI, and industry have led to the adoption of PR-EDB as the basis for an industry-wide data base by the EPRI Reactor Vessel Embrittlement Management Project. 


\subsection{Fracture Toughness Data}

The mechanical test results contained in PR-EDB and TR-EDB are Charpy impact test and tensile test data. The transition temperature approach, mainly relying on the Charpy impact test, is simple and has been used successfully. However, it has the limitation of being a temperature criterion rather than a more familiar stress criterion. Therefore, it cannot be used directly in the design. This shortcoming can be overcome by including fracture mechanics test results into EDB, which can offer a direct linkage to stress analysis and verify the relationships between the Charpy data and fracture toughness data. There are three categories of fracture toughness data available from the reports, static fracture toughness, $\mathrm{K}_{\mathrm{IC}}$ or $\mathrm{K}_{\mathrm{JC}}$, dynamics fracture toughness, $\mathrm{K}_{\mathrm{ID}}$, and arrested fracture toughness, $\mathrm{K}_{\mathrm{IA}}$ data. The general specimens used in fracture toughness experiments are Single Edge Notch Bending specimen (SEB), Compact Tension (CT), ArcShaped Tension (AT), Disk-Shaped Compact Specimen (DCT), Crack-Line-Wedge-Loaded Specimen (CLWL, or WOL), Double Cantilever Beam Specimen (DCB), and Center-Cracked Tension Panels (CCT). These specimens are carried out under different criteria and experimental procedures and are responsible for the particular kind of fracture toughness data. Therefore, it is necessary to separate the three major fracture toughness data and carefully distinguish the criteria among the different type specimens.

Considerable controversy still surrounds the question of upper-shelf fracture toughness, two methods are presently available for such determinations: the equivalent energy method and the $\mathrm{J}$ integral method. It was recommended that the equivalent energy method only be employed when the fracture of the specimen is cleavage controlled. From the available reports, the most frequently used fracture toughness specimens are CT, WOL, DCB and pre-crack Three Point Bend, therefore, the current main focus of specimens will be on these specimens. There are several schemes being used for the calculation of $\mathrm{J}_{\mathrm{IC}}$, among different reports, beside ASME's specification, these methods needed to be identified properly. Also there is needed to identify the different types of fracture behavior, and TAG as A-cleavage fracture, B-stable tearing but do not cross the $1.5 \mathrm{~mm}$ exclusion line with fast fracture at $\mathrm{P}_{\max }$, C-stable tearing with no cleavage, or D-stable tearing extend past the $1.5 \mathrm{~mm}$ exclusion line with cleavage thereafter. The index for validation of the test is also needed, for example, crack length (a) and specimen thickness (B) need to be consistent with the ASME's specification, a and B $>=2.5\left(\mathrm{~K}_{\mathrm{IC}} / \sigma_{\mathrm{ys}}\right)^{2}$.

The data obtained from different sources may have developed using different machines and different experimental specifications, the detailed description of the test procedures and testing apparatus, such as machine type and capacity, and responsible engineer, etc, need to be integrated into the data base. Those data will allow the user to trace back the original data source and pin down the difference among different experimental environment and testing methodology. There are several methods being used to determine the crack growth, such as these measured by Crack Mouth Opening Displacement (CMOD) method, Optical method, and Acoustic Emission Evaluation, or predicted by compliance method, and each may require a different adjustment depending on the application to the particular specimens, so this information needs to be handled carefully before registered into data base. For a dynamic or arrest fracture toughness experiment, in order to generate higher initial fracture toughness, $\mathrm{K}_{\mathrm{IC}}$, the CT or WOL specimens normally are added with starter such as embrittlement weld bead or duplex specimen. 
The additional modification among the specimen, such as pre-crack or side-grooves are also needed to be registered into data base.

Phase I of this project has been completed, three categories of the fracture toughness data available from the reports: static fracture toughness, $\mathrm{K}_{\mathrm{IC}}$ or $\mathrm{K}_{\mathrm{IC}}$; dynamic fracture toughness, $\mathrm{K}_{\mathrm{ID}}$ or $\mathrm{K}_{\mathrm{JD}}$; and arrested fracture toughness, $\mathrm{K}_{\mathrm{Ia}}$ data, had been integrated into EDB.

\subsection{The Data Contents of EDB}

Three major categories of data are included in the EDB, namely, pre-irradiation material history, irradiation environments, and mechanical test results, and contains the following types of data (Fig. 4):

1. Fluence $(\mathrm{E}>1.0 \mathrm{MeV}, \mathrm{E}>0.1 \mathrm{MeV}$, and dpa), irradiation time, and irradiation temperature for each irradiated capsule; or at material test reactors,

2. Charpy impact test results before and after irradiation, both for individual specimen and evaluation of transition temperature and upper-shelf energy;

3. Tensile test results before and after irradiation;

4. Fracture mechanics test results before and after irradiation;

5. Chemistry data for each material;

6. Pre-irradiation heat treatment;

7. Data concerning the fabrication of weld material;

8. Lead factor data; and

9. Data related to the determination of the pressure-temperature (P-T) limits.

Most irradiations were performed at the typical operating temperature of power reactors, around $550^{\circ} \mathrm{F}$, but enough information for other temperatures is available from the test reactor to investigate in detail the influence of irradiation temperature on embrittlement.

The user of the EDB is expected to have some familiarity with the dBASE philosophy and software to take full advantage of the information contained in the data base. It should be noted that the data in the EDB are taken directly from the quoted documents without any interpretation or evaluation. All numerical values are given in the units of the original documents. All data from any particular record in the EDB file are obtained only from the document quoted in REF_ID, except as noted. More than one record of the same quantities may be included in the files if different documents report different evaluations of the same data. For instance, several determinations of the chemistry of the same material may be performed or fluences may have been updated based on improved methodology or cross section data. It is left to the user to select or, perhaps, average the different values for the same quantity. Automated analysis of the raw data files in the EDB is not recommended; additional evaluation, selection, and unit conversion will be necessary whenever these data are to be used for critical investigations and as support for the analysis of reactor safety issues. The creation of evaluated data files for such purposes is being considered for future releases. In order to allow the user to streamline the raw data and to generate the evaluated data files more easily, and to be able to duplicate the routine functions on 
the update analysis, it is necessary to further develop the EDB-Utilities to incorporate the built-in macros and special routines to handle "what-if scenario" into analysis and selection procedures.

\subsection{Supplementary EDB Data Input}

The supplementary EDB data input for the irradiation environment are irradiation-time history, group fluence spectra, and dosimetry data, which are essential for the detailed study of the rate effect, spectrum effect, and further investigation of the damage efficiency and residual defects. The degree of embrittlement is conventionally correlated with fast neutron or with dpa, which are measures of the production rate of point defects. However, radiation effects are driven not by the total atoms displaced but by the small fraction of point defects that avoid annihilation by mutual recombination and are made available to form clusters or to be absorbed at sinks. Therefore, radiation effects are determined by the survival rate of point defects, not by their production rate. The survival rate of point defects (or defect availability) depends on several factors: (1) the temperature of the material, (2) the rate at which the fluence is accumulated, and (3) the energy of the neutrons causing the displacements. Higher temperatures, higher flux levels, and higher energy neutrons all enhance recombination effects and result in fewer final defects. Thus the rate of development of radiation embrittlement under different irradiation environments will not necessarily scale with fast fluence or with dpa unless the survival rate of point defects for each different irradiation also scale with the total defect production rate. Therefore, the above-mentioned supplementary radiation environment data are crucial for obtaining a better correlation parameter for the prediction of the radiation embrittlement of RPV steels.

\subsection{Dosimetry Database}

Currently, EDB Irradiation Environment contains the reactor list, irradiation time, irradiation temperature from melt wires, and the final evaluated neutronic data, such as fluence and fluence rate. There is no detailed information about transport analysis, dosimetry results, and reactor operating history in the EDB Irradiation Environment. In order to have complete information on the irradiation environment for archive purposes and for future reevaluation of neutronic data for plant specific cases, such as fluence and lead factors, an additional data base, which covers the dosimetry results and neutronic analysis, is desirable, and is suggested to be created and integrated into EDB. Therefore, a new databse, "Dosimetry Data Base," is recommended to be developed and it will contain the following information;

a) Dosimetry results from each passive neutron monitor, such as measured activity, adjusted saturated activity, measured and adjusted calculated reaction rate, and detailed location of each monitor and the corresponding material type of surveillance specimens, such as plate or weld, or heat-affected-zone material. Also, the physical characteristics of the monitors should be included.

b) The Power-Time history of the reactor for each surveillance capsule.

c) A detailed specification of reactor geometry including surveillance capsule locations. 
d) A complete history of reactor core configurations.

e) The calculated neutron energy spectrum at the monitor location, and the calculated reaction cross section at the monitor.

f) The calculated neutron energy spectrum at the inner wall, and the $1 / 4 \mathrm{~T}, 1 / 2 \mathrm{~T}$, and $3 / 4 \mathrm{~T}$ thickness vessel wall locations.

g) Descriptions of transport code and methodology used in the neutron transport analysis and the adjustment analysis.

h) Calculated spectrum-averaged reaction cross sections at the center of surveillance capsules.

Another important category of mechanical test data for future EDB data input is the nondestructive test data, including the indentation test and ultrasonic test data. This proposed nondestructive data base is essential for the calibration and construction of the correlation between the existing mechanical test data and the nondestructive test results. This data may prove to be useful in the near future, especially for those older plants on the verge of exhausting all the available mechanical test specimens loaded in the surveillance capsules.

\subsection{Applications of EDB in Radiation Embrittlement of RPV Materials}

EDB uses in evaluating radiation embrittlement of reactor pressure vessels cover the following:

(1) Validation of the embrittlement prediction models.

(2) Parameter study of the chemistry compositions effect on radiation embrittlement.

(3) Irradiation temperature effect on radiation embrittlement.

(4) Investigation of the spectrum effect and rate effect.

(5) Characterization of heat treatment on radiation embrittlement.

(6) Study the defect stability by thermal annealing data.

(7) Correlation between Charpy shift and the increase in the tensile yield stress, and Charpy USE vs. tensile strength.

(8) Comparison and unification of Raw Charpy data fitting procedures.

(9) Statistical Characterization of Radiation Embrittlement.

Research had been done on, but not limit to, the following issues:

(1) Analyses of correlation monitor materials, A302B and A533B, in surveillance capsule of commercial power reactors.

(2) Sensitivity of specimen orientation to radiation embrittlement.

(3) The phosphorus effect in low copper materials.

(4) Check if test results from LT specimens reduced to $65 \%$ of their value provided conservative estimates of the expected value from TL specimens.

(5) Comparison of the current PR-EDB data base with Reg. Guide 1.99 Rev. 2's data base.

(6) Re-evaluation of the two standard deviation values for the base and weld materials.

(7) Comparison of Reg. Guide 1.99 Rev. 2's model with French FIM's model for the prediction of the radiation embrittlement.

(8) Raw Charpy data curve fitting procedures. 
(9) Comparison of TANH fitting program at ORNL with one from the EPRI.

(10) Comparison of PR-EDB with EG\&G's data base on SHSS02 correlation monitor materials.

(11) Metallurgical and irradiation effects study for the Yankee Rowe's reactor related events.

(12) Data analysis for evaluation of irradiation temperature effects.

(13) Thermal annealing study on radiation embrittlement.

(14) Verification of initial RT $\mathrm{NDT}_{\mathrm{T}}$ and ART per ASME Code and R.G. 1.99, Rev. 2.

(15) Determination of Pressure-Temperature Limits curves for commercial power reactors.

(16) Background study for R.G. 1.99, Rev. 2.

(17) Residual study for the issue regarding the improvement of fluence evaluation per Rev. 2 of R.G. 1.99.

(18) Development of power reactor embrittlement prediction models. 


\section{EDB ARCHITECTURE}

The methodology and key issue concerns for developing EDB database are:

a) generic and versatile data structures allow easy data integration and future data expansion.

b) retain the data information extracted from the sources

c) database consists of groups of small data files "connected" with "key identifiers"

d) embed QA protocol into data structure development and routinely data input

e) provide good data linking mechanism to allow data easily integrated into Xbase program for developing automated program library for update and routine analysis.

\subsection{EDB Structure Design Criteria}

Most data collections are composed of one or more tables that are two-dimensional arrangements with rows called "records" and the columns designated as "fields." Each record represents an individual (person, test, reactor, etc.), and each field, a particular attribute. For example, a table for persons may list name, age, height, weight, etc., each in its designated field. A data base that consists of only one table is called "flat," and this is the format that was used for the older collections, including the MPC Data Base. This approach makes data retrieval easy, requiring no special software, but ignores completely any connections that may exist between different types of data. For instance, chemistry data are common to a particular material and must be repeated for every record that contains the same material. Also, more than one chemistry determination may be made for the same material such that not only the same chemistry is repeated over several records, but each record must provide room for several different chemistries. (This was actually the case in the MPC Data Base.) These requirements make a flat data base unwieldy and error prone and has led to the introduction of "relational" data bases.

In a relational data base the information is split into several different tables (files), each of which contains only related data. Data from different files are connected (related) with each other by means of "Unique Identifiers" that are common to the related tables. For instance, all chemistry data are collected in a chemistry file, where each chemistry record contains a unique material identifier. The same material identifier is contained in each test record for that material so that test results can be combined with chemistry. In this way duplications are avoided, and it is also possible to list any number of different chemistry determinations for the same material. The downside is that several files must be linked together to extract the desired information, and considerable software support is necessary to do this effectively. Such software support is now widely available as is the use of relational data bases for all but the very simplest data base applications. For the Embrittlement Data Base, both PR-EDB and TR-EDB, the relational data base format has the following significant advantages:

To avoid the problems of a single table data base, the EDB is designed as a collection of many different data files, each of which closely resembles the data tables found in the original surveillance reports. For instance, most reports have tables containing transition temperatures 
and upper shelf energies for Charpy specimens before and after irradiation and/or the shift in these values during irradiation, and these data are collected in the file "SHFT_PR". Data are collected as reported (i.e., there are fields for unirradiated, irradiated, and shift values, depending on what is reported; fields are added for units because some reports use U.S. units, others, the new international units, and still others, the older European engineering units (see Table 1).

Similarly, information about tensile tests is collected in the file TEN_PR, information about the irradiation, such as capsule fluence and temperature, in the file REAC_PR, and so on. This approach has many significant advantages:

1. The structure of the data files needs not be predetermined; the data files are designed according to what is available in the original reports, and new data files can be added without disturbing the existing ones.

2. Because every record in a data file originates from a single report and, in most cases, from a single table in this report, a unique reference, including page number(s), can be given for each record.

3. Multiple determinations of the same quantity are given in different records, each with its proper references. Such multiple determinations occur, for instance, if the chemistry is determined by the manufacturer of the material as well as from broken specimens. Also, fluence determinations are frequently updated in subsequent reports using improved neutron physics calculations. All different determinations are kept in the EDB, and it is up to the user to decide which determination to use for a particular application or, perhaps, calculate averages from several of them.

Because the data in the EDB are distributed over many different files, means must be provided for combining data from several files. This is done with the technique of "relational data bases," specifically dBASE by Ashton Tate. The format for data and auxiliary files and the techniques introduced by dBASE are now widely used in data base applications. The choice of the dBASE format for the EDB allows the use of any version of the dBASE software as well as any of the other software packages which use the dBASE format, such as FoxPro and MS-ACCESS. The linkage between different data files in a relational data base is provided by "key identifiers" which are common to these files. For instance, all files with data concerning a specific material such as results of material property tests, material manufacture, heat treatment, and chemistry contain a field for the material identifier HEAT_ID. Similarly, files with data concerning irradiations contain fields for the identifiers of the reactor, PLANT_ID, and the surveillance capsule, CAPSULE. Extreme care must be exercised to assign the correct identifier to each record since otherwise connection between data from different records cannot be made or are made incorrectly. To assure correct identifications, numerous cross checks are made which have the additional advantage that many mistakes are caught in this manner that have eluded conventional proofreading.

The best designed data base is useless without convenient software to extract data for any given application. The dBASE-compatible software provides the necessary tools but requires some expertise from the user for successful application. For this reason, EDB-Utility has been written 
to perform many processing steps such as retrieval and selection of data, calculations, and display to the screen or printer. This software is menu-driven so that it can be used without

Table 1 Units used in Embrittlement Database (EDB) files

\begin{tabular}{|c|c|c|c|c|c|c|}
\hline \multirow{2}{*}{ Description } & \multirow{2}{*}{$\begin{array}{l}\text { Symbol used } \\
\text { in EDB }\end{array}$} & \multirow{2}{*}{ Type of unit } & \multicolumn{4}{|c|}{ Conversion factors } \\
\hline & & & \multicolumn{2}{|c|}{ to English units } & \multicolumn{2}{|c|}{ to SI units } \\
\hline Mil (1/1000 inch) & mil & Length & 0.001 & inch & 0.00254 & $\mathrm{~cm}$ \\
\hline Millimeter & $\mathrm{mm}$ & Length & 0.03937 & inch & 0.1 & $\mathrm{~cm}$ \\
\hline Centimeter & $\mathrm{cm}$ & Length & 0.3937 & inch & 1.0 & $\mathrm{~cm}$ \\
\hline Inch & in. & Length & 1.0 & inch & 2.54 & $\mathrm{~cm}$ \\
\hline Fahrenheit & $\mathrm{F}$ & Temperature & 1.0 & $\mathrm{~F}$ & $(F-32) / 1.8$ & $\mathrm{C}$ \\
\hline Centigrade & $\mathrm{C}$ & Temperature & $1.8 C+32$ & $\mathrm{~F}$ & 1.0 & $\mathrm{C}$ \\
\hline Kelvin & $\mathrm{K}$ & Temperature & $1.8 \mathrm{~K}-460$ & $\mathrm{~F}$ & $1.0 \mathrm{~K}-273$ & $\mathrm{C}$ \\
\hline Foot-pounds & $\mathrm{ft}-\mathrm{lb}$ & Energy & 1.0 & $\mathrm{ft}-\mathrm{lb}$ & 1.3558 & joules \\
\hline Joules & $\mathrm{J}$ & Energy & 0.7376 & $\mathrm{ft}-\mathrm{lb}$ & 1.0 & joules \\
\hline Kilogram-meters & kgm & Energy & 7.2330 & $\mathrm{ft}-\mathrm{lb}$ & 9.8066 & joules \\
\hline $\mathrm{Mkp}^{a}$ & MKP & Energy & 7.2330 & $\mathrm{ft}-\mathrm{lb}$ & 9.8066 & joules \\
\hline $\mathrm{Mkp} / \mathrm{cm}^{2}{ }^{b}$ & MK/C2 & Energy & 5.78 & $\mathrm{ft}-\mathrm{lb}$ & 7.92 & joules \\
\hline $\mathrm{KJ} / \mathrm{m}^{2}$ & $\mathrm{KJ} / \mathrm{m}^{2}$ & Energy & & & 1.0 & $\mathrm{KJ} / \mathrm{m}^{2}$ \\
\hline Kilogram/mm² & $\mathrm{K} / \mathrm{MM}$ & Stress & 1.4223 & ksi & 9.8066 & $\mathrm{MPa}^{c}$ \\
\hline Kip/inch ${ }^{2}$ & KSI & Stress & 1.0 & ksi & 6.895 & $\mathrm{MPa}^{c}$ \\
\hline Pound/inch ${ }^{2}$ & PSI & Stress & 0.001 & ksi & 6.895 & $\mathrm{kPa}^{\mathrm{c}}$ \\
\hline Mega Pascal & $\mathrm{MPa}$ & Stress & 0.145 & ksi & 1.0 & $\mathrm{MPa}^{c}$ \\
\hline $\mathrm{MPa} \sqrt{\mathrm{m}}$ & $\mathrm{MPa} \cdot \mathrm{m}^{\wedge}(1 / 2)$ & Stress & 0.910 & ksi $\sqrt{ }$ in & 1.0 & $\mathrm{MPa} \sqrt{\mathrm{m}}$ \\
\hline Ksi $\sqrt{i n}$ & KSI $\cdot \mathrm{in}^{\wedge}(1 / 2)$ & Stress & 1.0 & ksi $\sqrt{ }$ in & 1.099 & $\mathrm{MPa} \sqrt{\mathrm{m}}$ \\
\hline Second & $\mathrm{S}$ & Time & 1.0 & $\mathrm{~s}$ & 1.0 & $\mathrm{~s}$ \\
\hline Hour & $\mathrm{H}$ & Time & 3,600 & $\mathrm{~s}$ & 3,600 & $\mathrm{~s}$ \\
\hline Day & $\mathrm{D}$ & Time & 86,400 & $\mathrm{~s}$ & 86,400 & $\mathrm{~s}$ \\
\hline Year (365 Days) & Y & Time & $31,536,000$ & $\mathrm{~s}$ & $31,536,000$ & s \\
\hline \multicolumn{7}{|c|}{$\begin{array}{l}{ }^{a} \text { Same as kilogram-meters. } \\
{ }^{b} \text { Energy relative to the cross section of the Charpy minus notch }=0.80 \mathrm{~cm}^{2} \text { (reported for the Beznat } \\
\text { reactors). } \\
{ }^{c} \mathrm{kPA}=\text { kilo Pascal }=10^{3} \text { Pascal; } \mathrm{MPa}=\text { Mega Pascal }=10^{6} \text { Pascal. }\end{array}$} \\
\hline
\end{tabular}

extensive training. An overview of the data flow in the PR-EDB is given in Fig. 6. The source data are first transcribed to "Raw Data Files" in dBASE format as faithfully as possible with complete references. Data entry is currently done through the keyboard. Direct transfer will be used whenever computer-readable documents are available. Any deviation from the norm, which was either reported or noted during transcription (such as the correction of obvious typographical errors) is indicated in the NOTES field. Data from every available report are included, except when the information in a later report is simply a duplication of earlier data without any changes. The dBASE format distinguishes between "character," "numerical," and "date" fields, but all data in the PR-EDB are entered as characters. This somewhat complicates the numerical manipulation of data that have to be converted to numerical form first, but gives much greater freedom to data entry. Information such as $<0.001$ for an upper bound or RT for room temperature can be copied from the original report without change. Missing information is always indicated by a blank data field, which is not possible in numerical or date formats. The 
Raw Data Files are next converted to processed files by the EDB-Utilities or other user-supplied software. Processed files will typically be in dBASE format, but ASCII-coded files can also be obtained from dBASE-compatible software for input into scientific software (e.g., numerical analysis programs written in FORTRAN), word processors, and spreadsheets. With the additional software, tables and graphs can be created for the purpose of model fitting, model verification, and other applications.

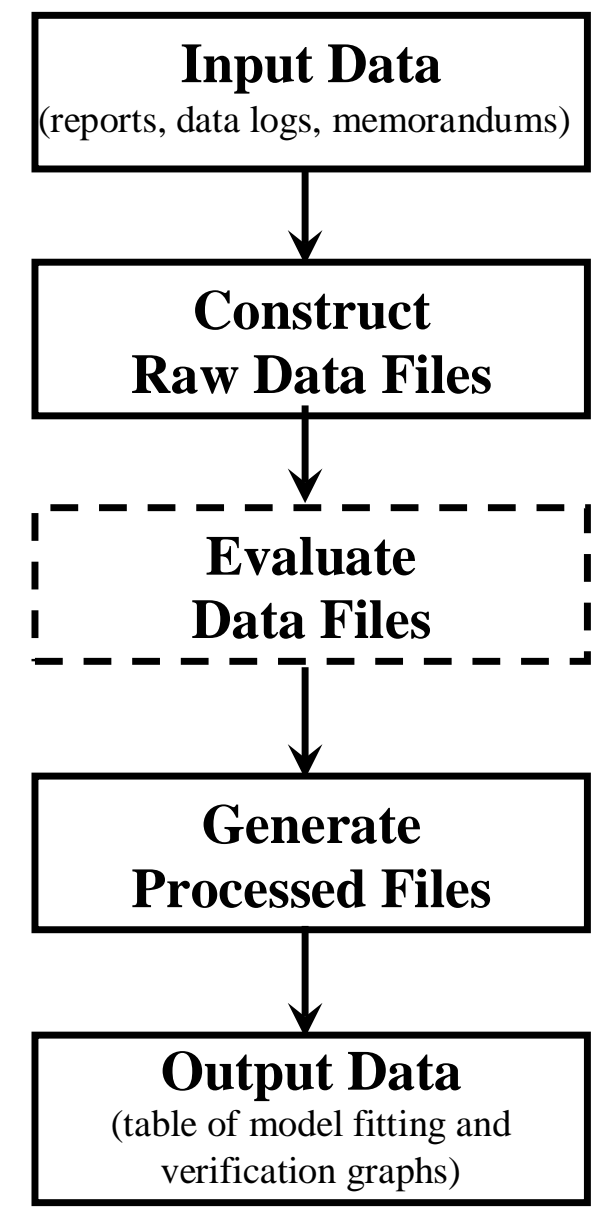

Figure 6 An overview of the data flow in the EDB

\subsection{Key Identifiers}

One or more fields in each data file are occupied by "key identifiers," which provide the means for combining data from several files through "relations" that link the corresponding records in these files. These fields differ from other data fields in that the key identifiers are assigned by the manager of the data base in a manner that the same unique identifiers are used to label the data among the various data files that refer to the same experiments, materials, or source 
documents. The identifiers used in the reports do not always provide such unambiguous labelling. The following key identifiers are used in the current version of the PR-EDB:

\section{PLANT_ID}

Up to six characters are used to identify the reactor in which the irradiation was performed. Only up to three characters are used at present for the identification of commercial power reactors. A list of these identifiers with full name and other information about the reactor is given in the file REAC_LST.dbf. The reactor code in PLANT_ID is also used to identify the surveillance program as a source of data, even if these data do not refer specifically to irradiation (as in files about chemistry or heat treatment).

\section{CAPSULE}

Up to six characters are used to identify the surveillance capsule. These are mostly identical to the identifications used in the surveillance program. Sometimes specimens are lumped together which come from different capsules with similar fluences, and in these cases special identifiers for data sets from combined capsules are assigned.

\section{HEAT_ID}

The material identifier can have up to ten characters. A simple scheme was devised which works as follows: The coding used for the PR-EDB assigns the first letter to the material type, namely P_late, F_orging, W_eldment, H_eat-affected-zone material, or S_tandard reference material. The next $3+2$ letters contain the reactor identifier (first three letters of PLANT_ID) plus some identification number, if more than one material is used in the same reactor (e.g., 01, 02, etc; if practical, some correspondence between the EDB and report identifications is retained, e.g., PCC101, PCC102, and PCC103 stand for D7206-1, D7206-2, and D7206-3, respectively). For standard reference materials that are not restricted to a particular reactor, other identification letters are used, namely, SASTM for the A302B ASTM reference plate and SHSS02 for the HSST plate 02. The last four letters are reserved to distinguish between different parts of the same material (e.g., between surface and 1/2T, or between several sections of a plate) if different parts show markedly different material properties as documented in the reports. For instance, SASTM S1 and SASTM S2, denote pieces of the 6-in. ASTM A302B reference plate used in the Garigliano and Yankee Rowe reactors, respectively, whose baseline properties vary considerably from the same material used in Westinghouse reactors, which is denoted simply by SASTM. The file HEAT_LST.dbf gives a complete list of identifiers used in HEAT_ID together with the corresponding identifiers in the surveillance reports.

It was first considered to use the identifiers given in the reports for HEAT_ID, a practice used in the MPC Data base. This, however, has proven to be impractical. Base material (plates or forgings) is usually characterized by the heat number (assigned to the ingot) or a manufacturer's number (assigned to the plate or forging after fabrication; see, for instance, BAW-1820) and one or the other, or neither, is used in reports, frequently with different choices of identification in different reports. For welds, a weld code such as SA-1585 or WF-232 is sometimes used, but the same code may be applied to different welds of the same type (BAW-1820). Alternatively, the 
wire heat number has been used for identification or just the heat numbers of the two plates joined by the weld. A distinct identification is rarely given for the heat-affected-zone material.

\section{SPEC_ORI}

Different orientations of the material test specimens may lead to substantially different property test results, thus this identifier is needed to correctly link the properties of irradiated specimens to the corresponding baseline values.

Orientations are assigned in the now customary T-L-S system as described in ASTM Standard E399, with L the primary rolling or forging direction, or, for welds and HAZ, the direction of the weld seam; $T$ is perpendicular to $\mathrm{L}$ and parallel to the plate surface, and $\mathrm{S}$ is perpendicular to the plate surface. The first letter describes the longitudinal direction of the specimen (perpendicular to the crack surface, if any), and the second letter describes the direction of the crack propagation (perpendicular to the notch). The orientation for each specimen set was determined as well as possible, preferably from drawings, making sure that the same orientations are assigned to corresponding specimen sets. SPEC_ORI is left blank if no information is available.

\section{REF_ID}

In most files, each record is assigned a reference which indicates the source of the data. This is done by means of a 20-character field REF_ID. This identifier is usually a report number or a similar code which links it uniquely to the complete bibliographic information, i.e., author, title, and time of publication, which is given in the file REF_TITL.dbf.

\subsection{Correlation Monitor Materials (CMMs)}

Generally, the surveillance programs of commercial power reactors include CMMs in addition to base, weld, and HAZ of actual pressure vessel steels. These are specimens fabricated from standard reference steel plates that are similar in most cases in composition and heat treatment to the base material in the respective RPV and are supposed to serve as a reference for comparing the radiation embrittlement of the plant-specific material to the reference material and to detect anomalies in the radiation environment of the surveillance capsules. In order to use these materials as a reference, the "normal" behavior in regard to fluence, flux, and irradiation temperature must be established first, and this requires the collection of data from a sufficient number of irradiations of the material at different irradiation environments, such as different flux and temperatures. Such data, obtained from irradiations in surveillance capsules and test reactors, are presently collected in the EDB, and its information is used for this study. The majority of surveillance programs use one or the other of two sources for the reference material, depending on the material in the pressure vessel. One is the 6-in.-thick ASTM A302 grade B (A302B) reference plate by U.S. Steel, and the other consists of three 12-in.-thick A533 grade B class 1 (A533B-1) plates fabricated by Lukens Steel and heat treated by Combustion Engineering (CE). These three plates were fabricated for the Heavy-Section Steel Technology (HSST) program in the late 1960s. Most surveillance data come from the second plate (HSST02); 
however, the first plate (HSST01) and third plate (HSTT03) are also used in surveillance capsules.

The results of Charpy V-notch impact tests for A302B and A533B1 Correlation Monitor Materials (CMM) listed in the surveillance power reactor data base (PR-EDB) and material test reactor data base (TR-EDB) have been analyzed. The shift of the transition temperature at $30 \mathrm{ft}-$ lb $\left(\mathrm{T}_{30}\right)$ is considered as the primary measure of radiation embrittlement in the analysis. The hyperbolic tangent fitting model and uncertainty of the fitting parameters for Charpy impact tests were also carried out to rule out other bias from the fitting curves. For the surveillance CMMs data, the transition temperature shifts at $30 \mathrm{ft}-\mathrm{lb}$ (delta $\mathrm{T}_{30}$ ) generally follow the predictions provided by Revision 2 of Regulatory Guide 1.99 (R.G. 199). Difference in capsule temperatures is a likely explanation for large deviations from RG.199 predictions. Deviations from the R.G. 199 predictions are correlated to similar deviations for the accompanying materials in the same capsules, but large random fluctuations prevent precise quantitative determination. There is significant scatter in the surveillance data, some of which may be attributed to variations from one specimen set to another, or inherit in Charpy V-notch testing. The major contributions to the uncertainty of the R.G. 199 prediction model, and the overall data scatter are from mechanical test results, chemical analysis, irradiation environments, fluence evaluation, and inhomogeneous material properties. Thus in order to improve the prediction model, control of the above-mentioned error sources needs to be improved. In general, the embrittlement behavior of both the A302B and A533B1 plate materials is similar. There is evidence for a fluence-rate effect in the CMMs data irradiated in test reactors; thus its implication on power reactor surveillance programs deserves special attention.

\subsection{Test Reactor Database}

The Test Reactor Embrittlement Data Base (TR-EDB) is a collection of results from irradiations in materials test reactors. It complements the Power Reactor Embrittlement Data Base (PREDB), whose data are restricted to the results from the analysis of surveillance capsules in commercial power reactors. The rationale behind this restriction was the assumption that the results of test reactor experiments may not be applicable to power reactors, and regulatory decisions could, therefore, be challenged if such data were included. For this very reason the embrittlement predictions in the Reg. Guide 1.99, Rev. 2 were based exclusively on power reactor data. However, test reactor experiments are able to cover a much wider range of materials and irradiation conditions that are needed to explore more fully a variety of models for the prediction of irradiation embrittlement. These data are also needed for the study of effects of annealing for life extension of reactor pressure vessels that are difficult to obtain from surveillance capsule results.

The scope and purpose of this program, which is sponsored by the Nuclear Regulatory Commission (NRC), can be summarized as follows:

(1) Compile and verify a comprehensive collection of data from test reactor irradiation experiments of pressure vessel materials from U.S. and foreign laboratories. 
(2) Provide software support for the use of the data base by furnishing programs and maintaining compatibility with commercially available software.

(3) Maintain compatibility with the PR-EDB.

(4) Maintain compatibility with U.S. and International Standards.

(5) Facilitate the exploration and verification of embrittlement prediction models.

(6) Facilitate the exploration and verification of the effects of annealing for pressure vessel life extension.

The architecture of the TR-EDB is fully compatible with that of the PR-EDB so that the data from both data bases can be easily merged, if desired. (Some overlap does occur; a few data from the PR-EDB appear also in the TR-EDB to make the latter self-consistent.) However, there are some differences; some changes are due to the differences between power reactor surveillance and test reactor experiments while others are improvements on the first design of the PR-EDB and will be beneficial to both data bases. None of these changes affect the data themselves and the ability to combine and select corresponding data from both data bases. These changes will also not prevent the use of the EDB software package provided for both versions of the EDB.

An important difference between surveillance capsule and test reactor experiments is the way the results are reported. A surveillance capsule report is part of a legal requirement that assures some degree of uniformity and consistency in its content. Test reactor experiments, on the other hand, are designed for specific investigations and the reports, particularly the older ones, present only the data that are deemed relevant to particular questions, without regard to their possible use in other research. This is quite understandable given the effort and cost involved in publications but results in incomplete information about the reported data sets; for instance, raw Charpy data are usually presented only as graphs, if at all. Additional difficulties arise whenever the results of the same experiment are reported in different publications without clearly indicating this fact. Frequently, different descriptive codes are used for the same material or experimental capsule, and a considerable amount of detective work is necessary to identify each single experiment or material. (Duplicate entries of this type have been identified in the original MPC Data Base.) For the reasons stated above, verification of the TR-EDB data is difficult and cannot be as thorough and comprehensive as in the PR-EDB. An additional problem is that laboratories and researchers responsible for the published data are often no longer available or cannot be funded for the considerable work involved for outside reviews. All data have been, of course, checked internally for correctness and consistency, and all unresolved problems are reported in the "NOTES." Every effort is being made to resolve discrepancies by contacting the original investigators.

It is important to remember that this structure can easily be extended to accommodate additional types of data without change in the existing structure. Most of the files have a counterpart in the PR-EDB with essentially the same set of fields, including field names. A few fields have been added that were not needed in the PR-EDB, such as EXP_ID, or to remove ambiguities, such as the addition of a field for nominal temperature which was previously listed under maximum or minimum temperature. 
The data that are included in the TR-EDB were originally generated by specific experiments or groups of experiments that were designed to answer some specific questions. Accordingly, choice of irradiation conditions, types of specimen investigated, evaluation methods, and related aspects differ considerably from one experiment to another, and these differences have to be considered in the use of the data. For that reason a key identifier EXP_ID has been added to every record in almost all data files. EXP_ID is not primarily intended for linking the information between two or more data files; it's main link is to give a detailed description of each experiment in the file E_LST_TR.dbf and the related table in this report. Note that the identifier PLANT_ID in the material files in the PR-EDB, such as HEAT_LST.dbf, CHEM_PR.dbf, etc., is replaced by EXP_ID in the corresponding files H_LST_TR.dbf, CHEM_TR.dbf. For test reactor experiments that were performed in support of a power reactor surveillance program, the PLANT_ID of the power reactor is used as EXP_ID. This policy will be extended to the PR-EDB in future releases. However, this key identifier is not presently used in the PR-EDB. The file REF_TR.dbf links references with experiments. Note that any given experiment is usually described in more than one reference and that the same reference may also report more than one experiment.

The details of architecture pf TR-EDB and its associated linking "Key Identifiers" are illustrated in Fig. 7.

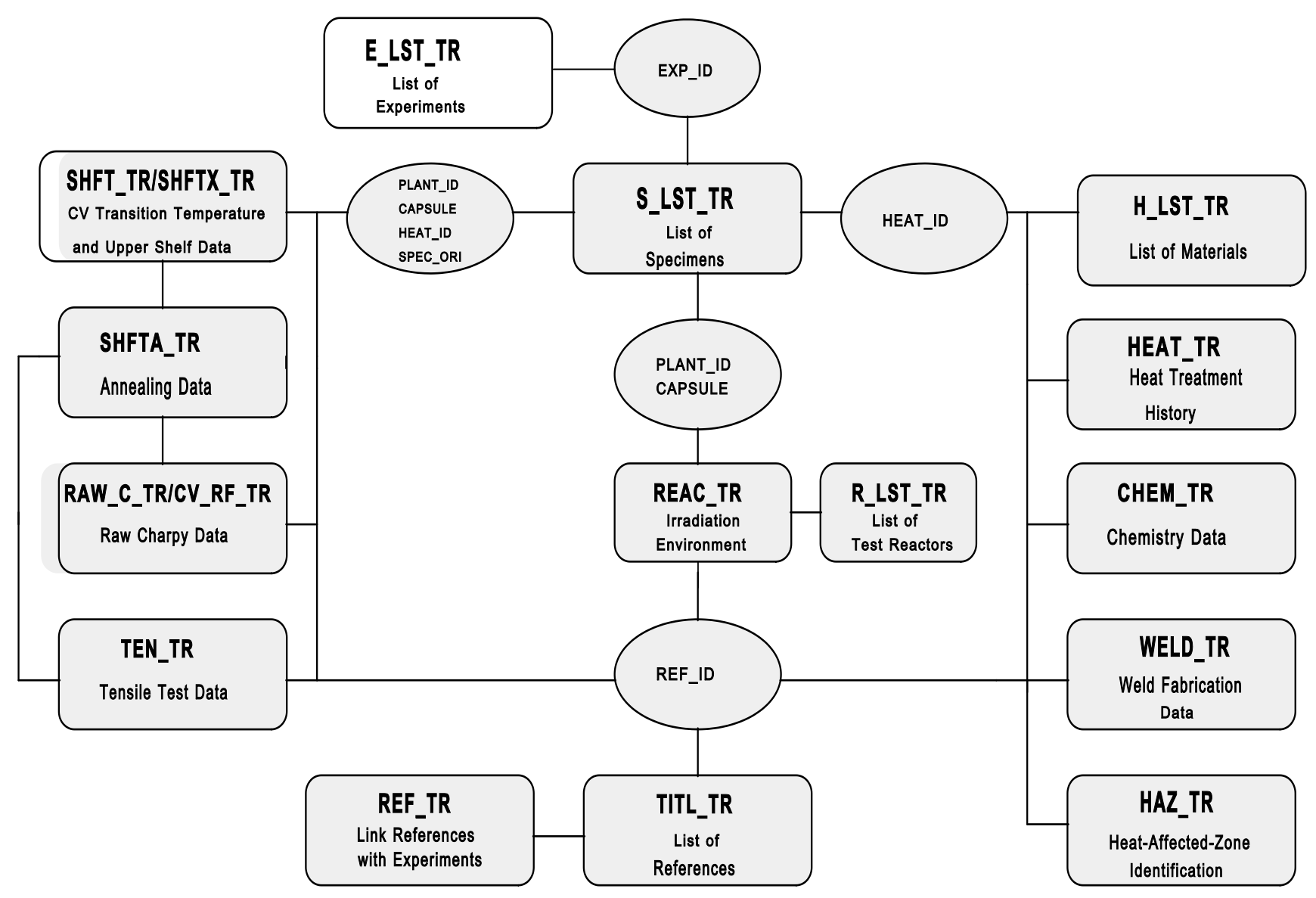

Figure 7 Architecture of TR-EDB 
The EDB data acquisition sheets contain 21 input data formats and the corresponding completion instructions. The samples of the data structure file and the data acquisition sheet are shown in Tables 2 and 3, respectively.

\section{Table 2 Data Structure for SPEC_GEO.dbf}

\begin{tabular}{|c|c|c|c|}
\hline \multicolumn{4}{|r|}{ Specimen Geometry } \\
\hline Field & Field_Name & Width & Description \\
\hline 1 & TAG & 1 & Used for Internal Operation \\
\hline 2 & EXP_ID & 6 & Experiment Identification, for test reactor data \\
\hline 3 & PLANT_ID & 6 & Reactor Identification \\
\hline 4 & HEAT_ID & 10 & Identification Code for given Material \\
\hline 5 & PROD_ID & 3 & Material Type \\
\hline 6 & SPEC_ORI & 2 & Specimen Orientation \\
\hline 7 & SPEC_POS & 4 & 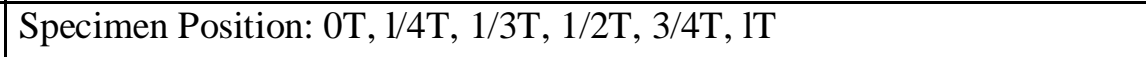 \\
\hline 8 & SPEC_TYPE & 6 & $\begin{array}{l}\text { Type of Specimen: S_tandard Charpy (CV), or M_initure CV, TEN_sile, } \\
1 \mathrm{~T} \text { C_ompact T_ension, 1/2T WOL, etc. for Non-standard specimen, } \\
\text { specimen thickness can be added in front of specimen type. }\end{array}$ \\
\hline 9 & GROOVE & 3 & \% of Specimen Side Groove, 20\%, 10\% etc. \\
\hline 10 & SPEC_WIDTH & 6 & Nominal Width of Specimen \\
\hline 11 & SPEC_WIDTT & 6 & Total Width of Specimen \\
\hline 12 & SPEC_HEGHT & 6 & Specimen Height or Specimen Length \\
\hline 13 & SPEC_THICK & 6 & Specimen Thickness or Specimen Diameter \\
\hline 14 & SPEC_NTHIC & 6 & Net Thickness of Specimen or Net Diameter of Specimen \\
\hline 15 & SPEC_UNIT & 4 & Unit Associated with Specimen Dimension \\
\hline 16 & REF_ID & 20 & Reference Identifier \\
\hline 17 & PAGES & 20 & Page Number \\
\hline 18 & NOTES & 30 & Pertinent Information Related to Data Entries \\
\hline
\end{tabular}


Table 3 Sample Data Acquisition Sheet

\section{General Information}

Component:

\section{Contributor:}

\begin{tabular}{||l|l|l||}
\hline Name: & Country: & State: \\
\hline City: & Street \& No: & Postcode: \\
\hline Contact person: Title: & Name: & Fax/Tel: \\
\hline
\end{tabular}

Remark contributor:

Modification: Yes $\square$

Steelmaker:

\begin{tabular}{||l|l|l||}
\hline \hline Name: & Country: & State: \\
\hline City: & Street \& No: & Postcode: \\
\hline
\end{tabular}

Remark steelmaker:

Component manufacture:

\begin{tabular}{||l|l|l||}
\hline \hline Name: & Country: & State: \\
\hline City: & Street \& No: & Postcode: \\
\hline
\end{tabular}

Remark component manufacture:

\section{Reactor:}

\begin{tabular}{||l|l|l||}
\hline \hline Name: & Country: & State: \\
\hline City: & Street \& No: & Postcode: \\
\hline Unit type: & Output (MWt): & Utility: \\
\hline Designer: & Architect: & RPV: \\
\hline RPV thickness(mm): & RPV ID(cm): & \\
\hline Operation date & year: month: day: & Operation temp. $\left({ }^{\circ} \mathrm{C}\right):$ \\
\hline
\end{tabular}

Remark reactor:

\section{Experiment Identification:}

\begin{tabular}{||l|l||}
\hline \hline Laboratory: & Authors: \\
\hline Publication date: & Ref. title: \\
\hline
\end{tabular}




\subsection{Annealing Database}

This database was developed to investigate the thermal annealing mechanism of aging RPV steels. There are 6 data files contained in this package, namely, HEAT_AN.dbf, HLST_AN.dbf, REAC_AN.dbf, WELD_AN.dbf, CHEM_AN.dbf and SHIFT_AN.dbf files. The first five data files have the identical data structure as in PR-EDB. (for example, HLST_AN.dbf can be referred to HEAT_LST.dbf of PR-EDB.)

The SHIFT_AN.dbf file contains the summary information of irradiation and annealing history. Each record only contains data for one annealing cycle, the additional annealing cycle (such as reannealing) will be listed as a separated record and connected by ANN_TAG, and the former cycle's IATT30 will be listed as UTT30 in the following annealing cycle. The ANN_TAG lists the annealing and irradiation history, and is consistent with the COND field (such as IARAR, etc), its basic terminology is explained as follows:

I: Pre-annealing irradiation

A: Annealing

R: Post-annealing irradiation

For example; PLANT_ID=UBR, HEAT_ID=WMEA8A, ANN_TAG=24121, COND=IARAR

The total annealing and irradiation history for IARAR condition is listed below;

I: five different pre-annealing irradiation fluences

A: seven different annealing temperatures, two different annealing durations

$\mathrm{R}$ : three different post-annealing irradiation fluences

A: two different re-annealing temperatures

$\mathrm{R}$ : two different post-re-annealing irradiation fluences

The ANN_TAG listed as 24121 means:

I_second pre-anneal irradiation fluence

A_fourth annealing condition

R_first post-annealing irradiation fluence

A_second re-annealing condition

R_first post-re-annealing irradiation fluence

The basic data structure of SHIFT_AN.dbf file is listed as follows:

TAG Used for Internal Operation

PLANT_ID Reactor Identification

CAPSULE Surveillance Capsule or Experiment Identification

HEAT_ID Identification Code for Given Material 
PROD_ID Material Type: P_late, F_orging, W_eld, HAZ, or SRM

SPEC_ORI Specimen Orientation: TL, LT, TS, etc.

CSP_F1 Fluence $>1 \mathrm{MeV}$ at Charpy Specimen Location $\left[\mathrm{n} / \mathrm{cm}^{2}\right]$

ANN_TAG Irra. and Anneal History Tag consistent with the COND Field

COND Irradiation and Annealing Condition

ANN_TEMP Annealing Temperature

ANN_HRS Duration of Annealing in Hours

PLANT_ID_R Reactor Identification for Re-irradiation

CAPSULE_R Capsule Identification for Re-irradiation

CSP_F1_R Fast Fluence of Re-irradiation at Charpy Specimen Location

UTT30 CVT at $30 \mathrm{ft}-\mathrm{lb}$, Unirradiated Charpy Specimen

ITT30 CVT at $30 \mathrm{ft}-\mathrm{lb}$, Irradiated Charpy Specimen

IATT30 CVT at $30 \mathrm{ft}-\mathrm{lb}$, After Annealing

IARTT30 CVT at $30 \mathrm{ft}-\mathrm{lb}$, After Annealing and Re-irradiation

UUSE Upper Shelf Energy, Unirradiated Charpy Specimen

IUSE Upper Shelf Energy, Irradiated Charpy Specimen

IAUSE Upper Shelf Energy, After Annealing

IARUSE Upper Shelf Energy, After Annealing and Re-irradiation

TEMP_U Unit used for Temperature Data

USE_U Unit used for Upper Shelf Energy Data

REF_ID Reference Identifier

PAGES Page Numbers

NOTES Pertinent Information Related to Data Entries, If Needed.

The annealing database structure illustrated the importance of using proper designed connector (in this case, ANN_TAG and COND) to integrate irradiation data from complex irradiation and thermal annealing cycles.

\subsection{P-T Database and the Associated Software PT_LIM}

The ability to resist fracture in a large steel pressure vessel containing the reactor core and its primary coolant constitutes an important factor in ensuring safety in the nuclear industry. The pressure-temperature (P-T) limits for light water reactors (LWR) stipulate a minimum coolant temperature that will ensure adequately high metal temperatures to preclude brittle fracture at any given pressure. The beltline region of the reactor pressure vessel (RPV) is the most critical region of the vessel because it is subjected to significant fast neutron exposure.

The P-T limits imposed on the reactor coolant system for all operating and testing conditions assure adequate safety margins against non-ductile or rapidly propagating failure, and are in conformance with the fracture toughness criteria of Appendix G of 10 CFR Part 50 and Section III, including Appendix G, "Protection Against Nonductile Failure", of the ASME Boiler and Pressure Vessel Code (ASME Code). 
The P-T Data Base for U. S. Power Reactors is a collection of data files in dBASE format that contains information about the geometry, and the irradiated and unirradiated properties of steel in RPVs. This data was collected directly from the power reactor surveillance reports. A computer program, P-T software, was also developed to determine the P-T limit curves using the simplified methods referenced in the ASME Code and WRC Bulletin 175. These methods have been verified to yield conservative values. This program is almost entirely menu-driven and is designed to allow a user not familiar with dBASE to process data, to perform calculations, and to plot data that are given in dBASE format.

The computer program, PT_LIM, has been written for a "DOS" - based personal computer to determine the pressure-temperature (P-T) limit curves using the simplified methods in the ASME Boiler and Pressure Vessel Code (ASME Code), Sect. III, Appendix G, "Protection Against Nonductile Failure." Fig. 8 provides the flow chart of the PT_LIM program development. The program is menu-driven and is designed with a built-in P-T data base. It also makes provisions for other user data.

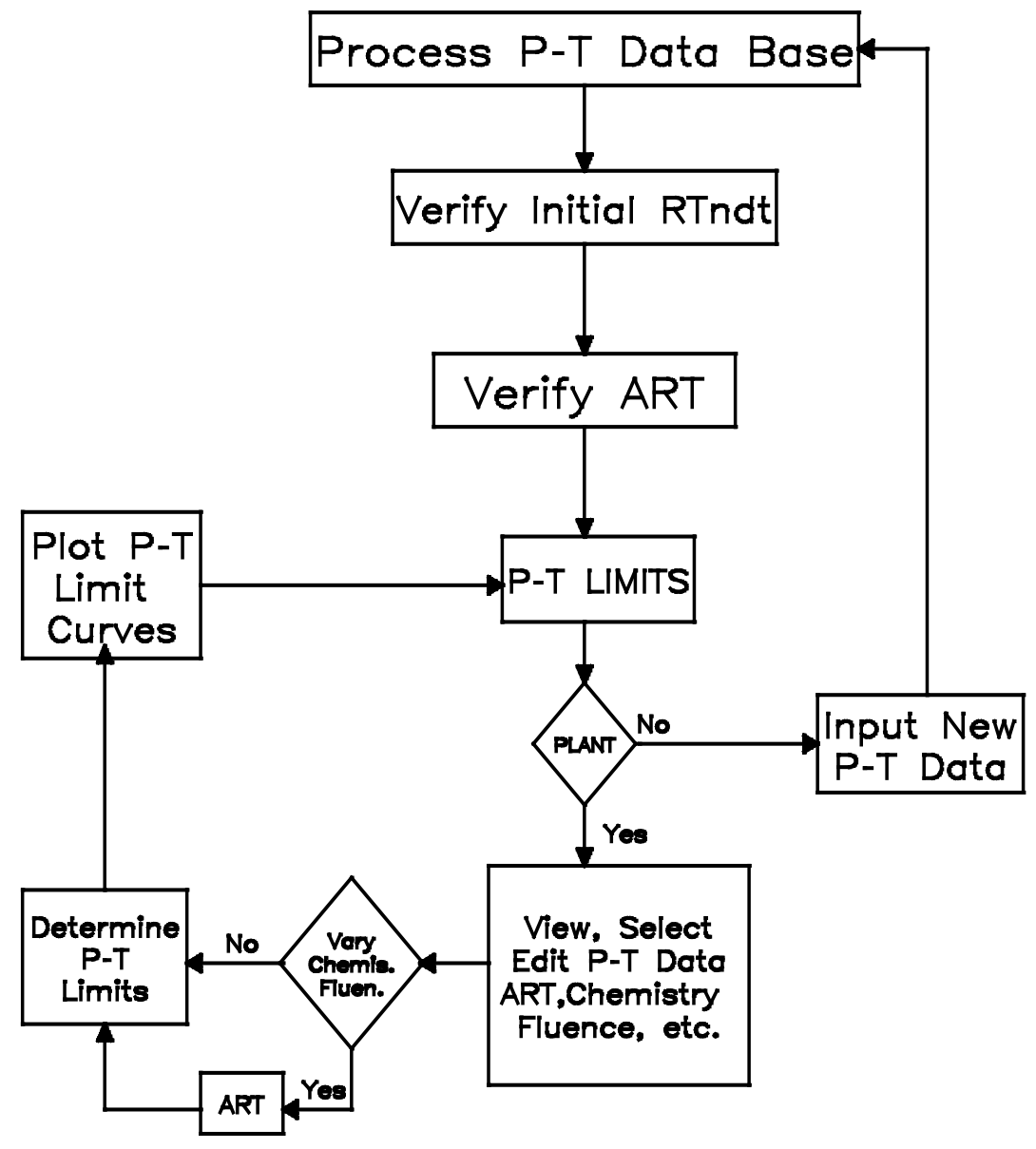

Figure 8 Functional flow of PT_LIM. 


\section{IAEA INTERNATIONAL DATABASE ON REACTOR PRESSURE VESSEL MATERIALS (IDRPVM)}

The author has been participated in IAEA IDRPVM database development and the description of this international aging database initiative is described below.

\subsection{IAEA IDRPVM Aging Database Initiative}

IAEA has developed a Lifetime Management of Nuclear Power Plants (LMNPP) program to facilitate the international exchange of information on the behavior of key components. A program on the reliability of reactor components has been established under the guidance of the International Working Group on the LMNPP (IWG-LMNPP). The main thrust of the reliability program is to advance the understanding of degradation mechanisms encountered in NPP operation and the initial focus of the program was the major degradation mechanism, irradiation embrittlement of the RPV, a key component which was thought to be irreplaceable and a plant lifetime limiting component The data base activity has therefore been prioritized starting with irradiation effects with activities planned to expand to other components such as materials for primary piping, steam generator, and containment.

It was recognized that data availability was a significant feature of LMNPP. It was also realized that in this key area of neutron irradiation effects on the properties of steels and welds that data bases already existed. These were broadly classified into two types data usually derived from accelerated irradiation in Materials Test Reactors (MTRs) or from materials surveillance programs in power reactors with some acceleration. Differences in behavior of these two types of irradiation have been observed. In addition it was a characteristic of these data bases that they related to the "national" compositions of the steels and welds, and the amount the data and information in the database varied significantly. The data bases were used to generate empirical relations which, in turn were used to predict irradiation response. Sometimes empirical guides have been used for materials outside the data base. A good example of the differences between such formulations is that between the US Regulatory Guide 1.99 (Rev 2) and the Russian Codes (which are similar to the French and Japanese Guides). The former guide uses the copper and nickel content to describe irradiation sensitivity while the latter includes copper and phosphorous to describe irradiation sensitivity. The Russian and Central European steels are different from the US, French or Japanese steels, but it is implicitly assumed that the irradiation and annealing response is the same for all these steels. There was a perceived need for a more comprehensive data base in order to identify and resolve some of these discrepancies and contribute to a greater understanding of this important effect.

\subsection{The Potential Benefits of IDRPVM Data Base}


The benefits accruing from an enlarged but international data base on neutron irradiation effects on RPV steels and welds are listed below:

a) improve the predictive capability of assessing remaining plant life of the RPV

b) improve the ability to schedule mitigation measures (preventive maintenance) in a timely way which may remove the need to disrupt plant operation.

c) enhance the assessment of degradation by a greater understanding of underlying metallurgical factors deriving from a large data base

d) provide the basis for improved descriptive Guides for international use

Specifically, this data base will help:

a) safety authorities in the preparation of improved design curves, reducing or decreasing conservatism but ensuring the maintenance of adequate and reliable safety margins in the field of licensing and safety report analyses.

b) designers in the provision of fuller descriptions underlying the design guides to improve the specification of materials and their use.

c) utilities in providing supportive data to that gained from surveillance experiments and provide data for remaining life assessment and mitigation activities

d) researchers to help provide better understanding of underlying mechanisms and in the provision of advice in the areas outside the data base

\subsection{Organization Mechanism for Developing IDRPVM Database}

\subsubsection{Organization of the Database}

There are two specific features of this database which influence the organization of the database, its membership and management. The data to be included is very expensive to develop having been generated in national and international programs on reactor materials. The requests from potential database members based on the above mentioned fact provide special conditions on the membership. Secondly, some data, especially that which provides a link between material properties and a particular utility or manufacturer, could have a commercial nature and therefore should be treated as confidential.

The database organization includes: IAEA, Database Custodian, Steering Committee and the database members.

The Role of the IAEA

The IAEA organizes the database according to requests from Member States and recognizes the importance of such activities as described below:

a. act as coordinator to compile, maintain and manage the database; 
b. manage the database to ensure that its rules and procedures are correctly and efficiently implemented taking into account the interests of all members in managing the operation of the database;

c. control access to the data through a system of confidentiality and data protection;

d. develop in consultation with database members, standards, formats, definitions, rules, procedures and guidelines to be used for the preparation and processing of input and for the creation and utilization of output;

e. prepare input of literature published by the IAEA and other UN organizations;

f. arrange meetings of the Steering Committee of the Database.

\section{The Role of the Custodian}

The Agency will identify and appoint the Database Custodian. The Custodian will act as the agent for the IAEA in operating and maintaining the database and providing an effective interface for member states and participating organizations. The Custodian will also arrange for data gathering from member states and database members, and will assist with data evaluation and distribution as appropriate.

\section{Steering Committee}

The Steering Committee consists of one representative from each member state of the International Database appointed by the national authority of the member state. Members of the Steering Committee shall elect from among themselves a chairman.

The responsibilities of the Committee are:

a. recommendations of the procedures for regulating the operation of the Database;

b. preparation of a progress report for the Agency's International Working Group on Lifetime Management of Nuclear Power Plants (IWG-LMNPP) meetings;

c. discussion of proposals from Members on the International Database.

Prior to the start-up of the Steering Committee, the ad-hoc group nominated by the International Working Group on Life Management of Nuclear Power Plants (IWG-LMNPP) shall perform the duties of the Steering Committee.

Database Members

The Database Members will include persons or organizations from Member States that provide and are entitled to use the database as well as to receive database information. Each Database Member will be responsible for data gathering, as well as validation and verification.

\subsubsection{Development Status of the Database}

The preparatory work of the Database includes:

a. development of the Database Specification;

b. development of the Database Agreement; 
c. preparation and distribution of official requests to Member States to join the Database;

d. expanding the Database to include degradation mechanisms other than irradiation and other components important to safety and reliability.

The document "International Database on Nuclear Power Plant Life Management -Database Specification has been published by the IAEA as -Working Material - IWG-LMNPP-95/4" in September 1995. This document provides information on objectives, scope, data collection and management requirements. The main part contains background information and information of generic interest, the appendices provide component specific requirements for some NPP components. In particular, Appendix A provides a detailed description of the International Database on Reactor Pressure Vessel materials. Appendix B describes Primary Piping Database Requirements for Plant Life Management, Appendix C addresses Steam Generators and Appendix D covers Containment.

The Division of Nuclear Power of the International Atomic Energy Agency (IAEA) in Vienna, Austria has supported neutron radiation effects information exchange through consultants meetings, specialists meetings, and conferences since the mid-1960s. Later, through an International Working Group (IWG) on Reliability of Reactor Pressure Components, information exchange and research activities were actively fostered through the Coordinated Research Program (CRP) sponsored by the IAEA. The final CRP meeting was held in November 1993 in Vienna where it was recommended that the IAEA should coordinate the development of an International Database on Reactor Pressure Vessel Material (IDRPVM) as the first step in generating an International Database on Aging Management.

\subsection{Comparison of IDRPVM and ORNL EDB}

\subsubsection{Comparison of Database}

A study was done by the author to provide special technical assistance to the NRC in monitoring and evaluating the IAEA activities in developing the IAEA IDRPVM, and to compare the IDRPVM with the Nuclear Regulatory Commission (NRC) - Oak Ridge National Laboratory (ORNL) Power Reactor Embrittlement Data Base (PR-EDB) and provide recommendations for improving PR-EDB. A first test version of the IDRPVM was distributed at the First Meeting of Liaison Officers to the IAEA IDRPVM, on November 1996. No power reactor surveillance data were included in this version; the testing data were mainly from CRP Phase III data. Therefore, because of insufficient data and lack of power reactor surveillance data received from the IAEA IDRPVM, the comparison is made based only on the structure of the IDRPVM. In general, the IDRPVM and the EDB have very similar data structure and data format.

One anticipates that because the IDRPVM data will be collected from so many different sources, quality assurance of the data will be a difficult task. The consistency of experimental results will 
be an important issue. A very wide spectrum of material characteristics of RPV steels and irradiation environments exists among the various countries. Hence the development of embrittlement prediction models will be a formidable task.

The IAEA's material aging data base contains 6 categories of data, namely; material history, aging history, mechanical testing, references, non-destructive testing, and collected curves. Currently, this data base refers only to reactor pressure vessel material surveillance (thermal and irradiation effects). The key identifier, "IAEA_CODE", serves as the only prime linking parameter through all the data files. The detailed contents of each category and its relation to PR-EDB will be discussed below, and its functional flow is illustrated in Fig. 9.

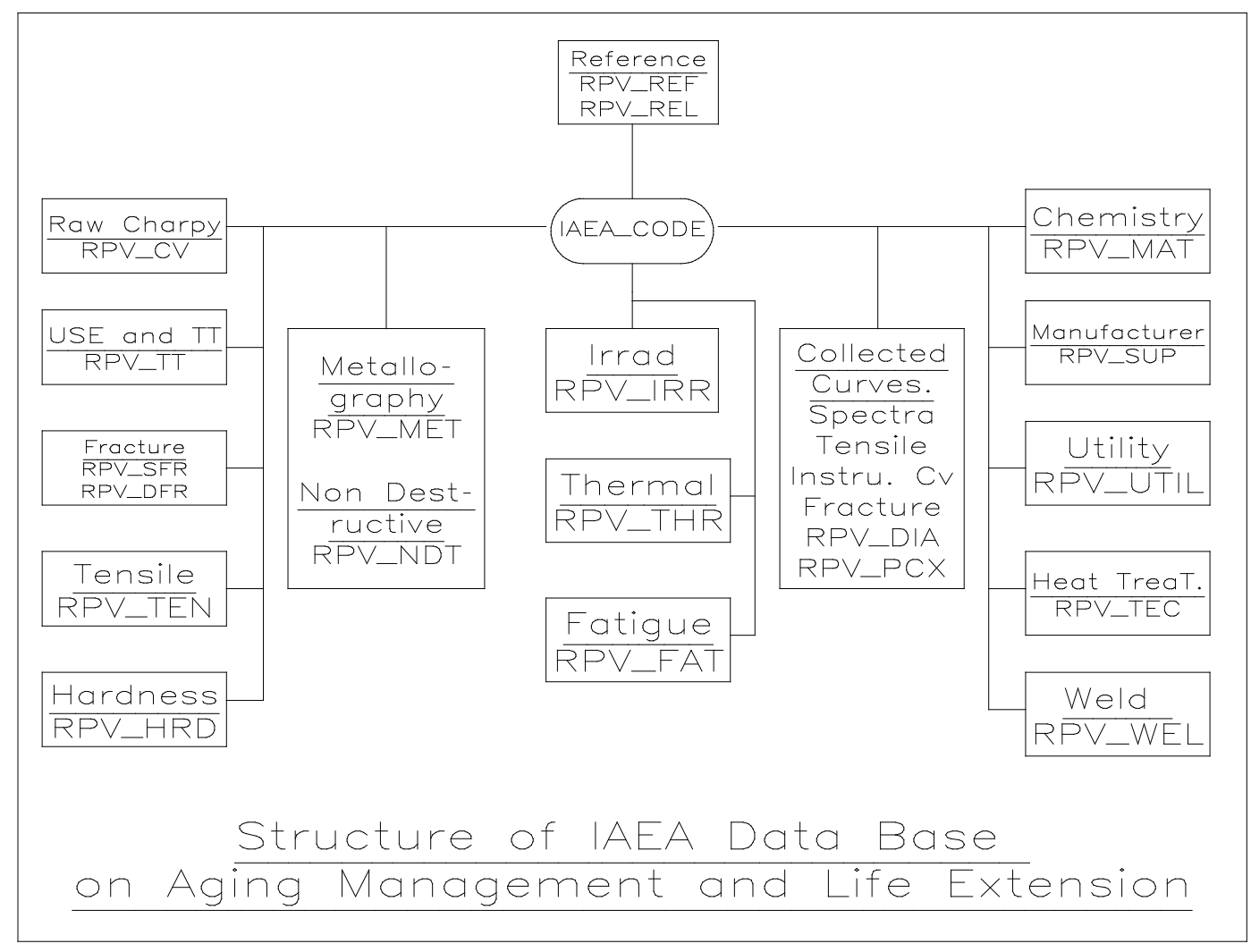

Figure 9 IAEA IDRPVM database structures

\section{Material History:}

A total of six data files were included in this category, namely; RPV_MAT, RPV_SUP, RPV_UTIL, RPV_TEC, RPV_WEL. The data contents of this category are very similar to that of PR-EDB. Where, RPV_MAT is equivalent to CHEM_PR; RPV_WEL is equivalent to WELD_PR plus weld heat treatment from HEAT_PR; RPV_TEC is equivalent to HEAT_PR; 
RPV_SUP is equivalent to HEAT_LST; and RPV_UTIL is equivalent to RLST_PR of PR-EDB. EDB has HAZ_PR data file, did not appear in IAEA.

\section{Mechanical Testing:}

This category includes four different types of mechanical testing, namely, Charpy, fracture, tensile, and hardness test. The data formats are almost identical to that of PR-EDB, except that the IAEA has system information embedded in the individual files, EDB intends to build a global system file to contain system information per EXP_ID, and IAEA did not contain the reported shift file, SHFT_PR.

Where, RPV_CV is equivalent to RAW_C_PR; RPV_TT is equivalent to PR-EDB's hyperbolic tangent fit data file, RAW_C_RS; RPV_TEN is equivalent to TEN_PR; RPV_SFR and RPV_DFR are equivalent to KIC and KID, respectively. Currently, there is no hardness data in PR-EDB, however, the data structure of RPV_HRD is very similar to the proposed hardness data file of PR-EDB.

\section{Aging History:}

Three types of data were included, namely, irradiation aging, thermal aging, and fatigue aging. Where, the RPV_IRR is equivalent to REAC_PR of PR-EDB, however, minor differences exist, such as fluence(E>0.5MeV), AVG_FLUX, MAX_FLUX, and LEAD_FACT contained in IAEA, whereas, uncertainty of the fluence, fluence $(\mathrm{E}>0.1 \mathrm{MeV})$, maximum and minimum capsule temperature, and effective power time, are contained in PR-EDB only. There are no fatigue aging data in PR-EDB, and with very limited thermal aging data in PR-EDB, however, thermal aging data structure, RPV_THR, is embedded in REAC_PR.

\section{References:}

This RPV_REF is equivalent REF_TITL in PR-EDB.

Non-destructive testing:

Data such as metallography, fractography, magnetic data, acoustic data, and x-ray's data, will included in this category. Currently, there is no activity on nondestructive data in PR-EDB.

\section{Collected Curves:}

Graphical data, such as instrumented Charpy test data, Static fracture test data, and flux distribution, spectra data, will be included in this category. Currently, only the instrumented Charpy's trace data is available in EDB.

\section{Conclusion}


The IDRPVM and the EDB have a very similar data structure. However, the drop weight test data, initial $\mathrm{RT}_{\mathrm{NDT}}$, and RPV geometry, are not contained in the IDRPVM database. These three items are needed for the determination of the pressure-temperature limits of operating reactors. Table 4 shows the comparison of EDB and IDRPVM databases architecture, including both existing files and proposed files.

One anticipates that since the data is collected from so many different sources, quality assurance of the data will be a difficult task. The consistency of experimental test results will be an important issue. A very wide spectrum of material characteristics of RPV steels and irradiation environments exists among the various countries. Hence the development of embrittlement prediction models will be formidable task.

Finally, it was noted that eight countries were present at the First Meeting of Liaison Officers to the IAEA IDRPVM. The U.S. did not receive an invitation due to a bureaucratic error at IAEA. The proposed inputs from the 8 countries are described in Section 5.5 and a summary of the data already listed in EDB was also provided to NRC. Based on the timetable for the receipt of data from the 8 countries, the first release of the IDRPVM by IAEA was November-December 1997. The second Liaison Officers’ Meeting was scheduled in February 1998.

Table 4 Comparison of EDB and IDRPVM

\begin{tabular}{|c|c|c|c|}
\hline GROUP DATA & SUBGROUP DATA & ORNL EDB & IAEA IDRPVM \\
\hline $\begin{array}{l}\text { Material } \\
\text { Identification }\end{array}$ & $\begin{array}{l}\text { - Material Chemistry } \\
\text { - Material Supplier } \\
\text { - Utility Supplying Data } \\
\text { - Base Material Technology } \\
\text { - Weld Material Technology } \\
\text { - HAZ Material Technology }\end{array}$ & $\begin{array}{l}\text { CHEM } \\
\text { HEAT_LST } \\
\text { REAC_LST } \\
\text { HEAT } \\
\text { WELD } \\
\text { HAZ }\end{array}$ & $\begin{array}{l}\text { RPV_MAT } \\
\text { RPV_GEN } \\
\text { RPV_UTIL } \\
\text { RPV_TEC } \\
\text { RPV_WEL }\end{array}$ \\
\hline Aging History & $\begin{array}{l}\text { - Irradiation Environment } \\
\text { • Thermal Aging Environment } \\
\end{array}$ & $\begin{array}{l}\text { REAC } \\
\text { REAC }\end{array}$ & $\begin{array}{l}\text { RPV_IRR } \\
\text { RPV_THR }\end{array}$ \\
\hline Mechanical Testing & $\begin{array}{l}\text { - Tensile Testing Results } \\
\text { - Charpy-V Impact Testing } \\
\text { - Static Fracture Testing } \\
\text { - Dynamic Fracture Testing } \\
\text { - Hardness Testing } \\
\text { - Transition Temperature \& } \\
\text { Hyperbolic Tangent Coefficients } \\
\text { - Parameters of Exponential Fit } \\
\text { for Fracture Toughness Data }\end{array}$ & $\begin{array}{l}\text { TENSILE } \\
\text { CHARPY } \\
\text { KIC, KJC } \\
\text { KID, KJD } \\
\text { HARDNESS } \\
\text { SHFT_CV \& } \\
\text { RAW_C_RS }\end{array}$ & $\begin{array}{l}\text { RPV_TEN } \\
\text { RPV_CV } \\
\text { RPV_SFR } \\
\text { RPV_DFR } \\
\text { RPV_HRD } \\
\text { RPV_TT } \\
\text { RPV_EXP }\end{array}$ \\
\hline References & $\begin{array}{l}\text { List of References } \\
\text { List of Related References }\end{array}$ & $\begin{array}{l}\text { REF_LST } \\
\text { REF_TITL }\end{array}$ & $\begin{array}{l}\text { RPV_REF } \\
\text { RPV_REL }\end{array}$ \\
\hline $\begin{array}{l}\text { Nondestructive } \\
\text { Testing }\end{array}$ & $\begin{array}{l}\text { Metallography Pictures } \\
\text { Other Nondestructive Test } \\
\text { Procedures }\end{array}$ & & $\begin{array}{l}\text { RPV_MET } \\
\text { RPV_NDT }\end{array}$ \\
\hline Collected Curves & $\begin{array}{l}\text { ASCII Diagrams of Data } \\
\text { Digitalized Pictures of Data }\end{array}$ & $\begin{array}{l}\text { Instrumented } \\
\text { Charpy data }\end{array}$ & $\begin{array}{l}\text { RPV_DIA } \\
\text { RPV_PIC }\end{array}$ \\
\hline
\end{tabular}




\subsubsection{Comparison of Database Software}

A test version of the IDRPVM software was distributed at the First Meeting of Liaison Officers, October 14-15, 1996, London, United Kingdom. The software was developed using Microsoft (MS) ACCESS. This first version only allows the users to select data, view or print data, and export data in MS ACCESS formats. The user is expected to develop a processing code to process and to analyze the data.

EDB on the other hand is a multi-function database management program that can retrieve, print, and export data, process data, plot data, and fit data. This code was developed before the release of MS Windows. Earlier EDB is designed for use with any personal computer using the DOS operating system. The data format that was chosen for EDB is dBASE (also adopted by IAEA). This format allows queries and data processing not only with the current dBASE software but also with any of the numerous "Xbase" developer tools, such as Clipper or Foxpro. The dBASE files can also be imported into most other databases, spreadsheets, and word processing programs that run in the DOS or WINDOWS environment. The more recent versions of these programs contain extensive utilities for generating reports including statistical, curve fitting, and graphic programs. For often-performed tasks a customized EDB Utility program was written based on Clipper and FORTRAN. This EDB-Utility is menu-driven requiring little or no training.

The data in the EDB are taken directly from the quoted documents (surveillance reports) without any interpretation or evaluation. All numerical values are given in the units of the original documents. If several determinations of chemistry are reported for the same material, it is left to the user to select or, perhaps, average the different values. Evaluation, selection, and unit conversion will be necessary whenever these data are to be used for investigations and analysis of reactor safety issues. The creation of evaluated data files is an integrated part of EDB program development. The software "Process Raw dBASE Files" was created for this purpose to generate a one to one correspondence between the key identifiers and its related information.

Many tasks, such as "to streamline the raw data and to investigate what-if scenarios," can be programmed and handled much easier in Windows environment than in DOS. Therefore, conversion of the EDB utility from DOS into Windows platform is the essential part of upgrading the EDB. The development in "Visual Object-Oriented Analysis and Design" in recent years provides a very powerful tool to generate a truly "User-Friendly" software for database management. Utilizing this tool to upgrade the EDB utility will speed up program development,

The proposed future EDB software has the following characteristic

- Operating under Windows environments;

- Retrieving, linking, and streamlining raw data to generate processed data files;

- Developing special routines to incorporate "what-if scenarios" into data selection procedures and analysis; 
- Built-in routines to automate the procedures for updating analysis, for follow-up studies, and updating report.

Currently, the Phase I of migrating EDB from DOS to Windows platform has been completed. The beta version was released to NRC during FY2006.

\subsection{RPV Surveillance Data Presented at "First Meeting of Liaison Officers to the IAEA IDRPVM Database."}

Participants from 8 countries were present at the meeting. Participants provided their proposed anticipated inputs into IDRPVM that were stated in the liaison minutes.

This section discusses what portions of the anticipated inputs are already listed in EDB, or CRP3 and Russian data that we had the databases already in our possession. However, the data format of CRP-3 did not allow the identification of whether the data is from a power reactor or a test reactor environment.

\section{Brazil input: Data from Angra I}

Currently, the data from capsule V of Angra I was listed in PR-EDB, including transition shift data and tensile data for 2 forgings, 1 weld and HAZ materials.

2. Hungary input: One NPP at the Paks site.

The NPP at Paks has 4 units (WWER-440.213) that currently are operated. Totals of 20 sets of specimens were withdrawn and test from those units. The new data as stated in Hungary inputs also includes the 0 level testing results, which are 900 Charpy results, 300 COD, 360 tensile results and 80 hyperbolic tangent fit results.

Currently, CRP-3 data base listed 110 Charpy test results from Hungary, where 39 data are irradiated at $275^{\circ} \mathrm{C}$ and $4.2 \times 10^{19} \mathrm{n} / \mathrm{cm}^{2}$ reported by Paks. The listed 5 tensile test results were irradiated at $4.2 \times 10^{19} \mathrm{n} / \mathrm{cm}^{2}$.

3. Italy input: One Latina Gas Graphite Vessel, Three power reactor (Trino, Corso, Garigliano)

The PR-EDB contained detailed information on RPV surveillance program for Garigliano, including 44 transition shift data points for forging, weld, HAZ and CMM materials. CRP-3 did not contain any data from Italy.

4. Korea input: 11 nuclear units including 9 PWRs and 2 PHWRs are currently operating. 
PR-EDB contained 6 Charpy shift data from Korea Unit 1. CRP-3 did not contain any data from Korea.

5. Russia input: 7 NPP units with WWER-1000 and 6 NPP units with WWER-440.

As stated in the minutes, surveillance specimen test results for WWER-1000 are available only for 4 sets: Novovoronezh NPP unit 5 - 2 sets, Kalinin NPP unit 1 - 1 set, Balakovo NPP unit $1-1$ set.

For WWER-440, surveillance programs are only available for Kola 3 and 4.

All the above mentioned data are listed in the Russian database, that is part of foreign reactor database, a branch of EDB.

6. Spain input: 9 NPPs with 18 capsules.

Currently 7 PWR surveillance data are collected, and nothing had been done regarding 2 BWRs' data from Spanish utilities as stated in the minutes.

PR-EDB has 3 capsules for Jose Cabrera, and baseline data for Almaraz II, Asco Unit 1, and Asco Unit 2.

7. Ukraine input: 10 units of WWER-1000, and 2 unit of WWER-440.

According to the minutes, presently, the information is already collected on 6 WWER-1000 power units at Zaporizhya NPP site. It is expected to complete the rest of reactors by March 1997.

Currently, the Russian database contains surveillance data for Zaporizhya unit 2 and 3, South Ukraine unit 1, Balakovo unit 1, and Rovno unit 1, 2 and 3.

8. UK input: 7 steel Magnox reactors from Magnox Electric, and surveillance database from BNFL.

Based on the minutes, the above UK surveillance databases are completed.

PR-EDB does not contain UK surveillance data. CRP-3 contains the following Charpy and tensile data from UK: 401 Charpy test results where 199 samples were irradiated at $290^{\circ} \mathrm{C}$, and 75 tensile test results where 40 samples were irradiated at $290^{\circ} \mathrm{C}$. 


\section{EDB DATABASE QA PROTOCOL}

\subsection{PR-EDB QA}

The first compilation of the U.S. Power Reactor Embrittlement Data Base (PR-EDB), Version 1, was completed in October 1989, and the documentation was published in June 1990. Subsequently in cooperation with the Electric Power Research Institute (EPRI), additional verification and quality assurance of the data were performed by the U.S. reactor vendors. A record-by-record check by the vendors removed some errors and ambiguities, and filled many gaps by providing data from additional documents and vendor records that were not previously available to the authors. Not all data have been verified by the reactor vendors; however, all data have been entered as reported in the quoted references. All data from the Allis-Chalmers reactors Elk River and LaCrosse are unverified. Also, data from two independent studies that were not under the vendor's control, namely, the review of fluence calculation in NUREG/CR-3319 by Hanford Engineering Development Laboratory and a report on Babcock \& Wilcox welds (BAW-1803) were not verified by Westinghouse. Several other reports containing data that could not be verified by the vendors are also noted. The changes to the first compilation and documentation have been incorporated into PR-EDB, Version 1, Revision 1 (April 1991).

The EDB software has also been upgraded since the last publication by removing some mistakes, by adding additional options in the graphic programs, and by making the program execution more reliable and less subject to crashes due to user errors. However, no changes in the structure and running of the programs were made. The data on the distribution diskette includes a run of the raw Charpy file with the Monte Carlo fitting program using 100 iterations. The output data file, RAW_C_RS.dbf, should not be used uncritically because the fits were generated without any human intervention and may be distorted by outliers, an insufficient number of data points, and large data scatter. Thus it is recommended to first review the graphic display of every fit for possible irregularities and, perhaps, remove outliers as described in the manual. By implementing these fitting procedures we are able to detect the input errors in the PR-EDB or detect the data error stated in the original reports, from visual inspection of the outliers or the unreasonable trend curve fitting. These fitting utilities have proven to be very useful in assisting the QA protocol, where data outliers were generally found to be data input error, and unreasonable fits also spot the data error from the original source documents.

The characteristics of EDB QA protocol can be described below.

(1) Traceability of all data is maintained by complete references including page numbers

(2) Data verification of the PR-EDB were performed internal to ORNL by checking against references and checking for any inconsistence; externally the correctness of the data were performed by the vendors who were responsible for the insertion and testing of the materials 
in the surveillance capsules. All changes, updates or corrections were documented into special files for future references

(3) Data verification of the TR-EDB was performed internally as stated above. External verification was not feasible unless original investigator was available.

(4) EDB utilities, such as TANH curve fitting utilities, provide additional means to check the accuracy of EDB data input through the curve fitting procedures, parameter studies, embrittlement analysis and the embrittlement model development.

\subsection{EDB Data Input Procedures}

EDB data input sources are from RPV surveillance reports, data logs, technical publications, and official memoranda related to RPV radiation embrittlement.

1. Collection of reports:

For PR-EDB the primary source of surveillance documents is the NRC that sends copies of surveillance reports to ORNL. However, this is not always the case. In order to identify the new or the not received surveillance reports, the author often need to hunt through the related technical documentations and then ask NRC to provide these reports. Recently author utilize NRC ADAMS to retrieve the new surveillance report which is also a very time consuming process to look through the numerous surveillance related documents and to download the needed reports. In many occasions, during the large file transfer (which is normally in image tiff file) the connection would abort and it will need to be restarted from beginning. The reference list of the surveillance report provides further information regarding the chemistry, heat treatment, etc., or additional or similar test results that are not provided in the original reports.

As for TR-EDB, the data are collected from all the available technical documentations, or through the bilateral agreement with other country authorized by NRC.

\section{Data Input Control:}

The data of EDB are abstracted from a limited number of collected documents into register notes and then stored in "holding files" which have the same structure as their corresponding EDB files in order to facilitate appending them to the EDB. In this way, the files are similar and therefore more manageable. As each document is processed, the control sheet for input to the EDB form is used so that no file is advertently overlooked and to provide a record of when the input, quality assurance, and addition to the EDB was completed. The first step is to assign and record a unique reference identifier on the form and compare the identifier to REF_TITLE to ensure that it has not been previously assigned. During this stage, the key identifiers, which are used as linkage between different data files, will be assigned to the new surveillance data, where the related baseline data, including chemical composition, material history from the earlier reports, will also be checked for consistence or updated information. 
As the input to each file is completed, it is noted by the operator. When all the files have been processed, a copy of the control sheet and a disk containing the files are turned over to the person responsible for internal QA or verification of the data. When the QA is completed, the EDB manager will merge and integrate the data into the EDB format. When more than one report is included in a set of holding files, a cover form listing the report numbers, release data, and comments is attached to the control sheets and disk.

3. Release of Holding Files

When the input of the holding files is completed, copy the files to a disk. If more than one report was used to generate the holding files, a special note will be attached to the disk to list all the associated reports. Upon the completion of internal QA, the EDB manager will integrate all the available holding files into a single EDB file, a total of 14 such data files from EDB structure format will be generated and this forms the Update Version of EDB.

\section{Verification of Quality Assurance Check by Vendors}

Version 1 of the EDB was released to vendors (Westinghouse, General Electric, Babcock\& Wilcox, and Combustion Engineering). In order to verify the accuracy of the data. The Electric Power Research Institute was placed in charge of distributing the data base to the vendors and initiating a quality assurance procedure. Upon receiving the feedback from the vendors, ORNL EDB program will then start to integrate these comments into EDB.

5. Verification of Quality Assurance Check by EDB Utility for Data Processing

EDB utility provides a good means to carry out additional QA protocol during the data processing for Charpy trend curve development or parameter study for embrittlement analysis. If the errors of input data were not found through the general QA protocol, the data processing utilizing EDB utility normally can detect such data error attributed to unreasonable fit or outlier in the trend curve. And in many cases the causes of the error are identified and traced back to the error stated in the source documents. 


\section{GENERAL CONSIDERATION OF MATERIAL DATABASE QA PROTOCOL}

Data quality is a determinant of the success of any material information system. The information collected by the reactor designer needs to be complete and accurate if the system is to have a positive impact on this important project. A strategic review of the registry for the measurements of data quality control compared among similar systems for reactor safety surveillance is recommended. However based on our experience, while a number of data quality assurance activities were undertaken by the data registry staff, these procedures had not been organized into a data quality assurance protocol. It is important to carry out a thorough strategic review plan that such a protocol be prepared, implemented and evaluated on an ongoing basis forming a data quality assurance system.

\subsection{Data Quality Definitions}

For the purpose of evaluating reactor safety surveillance systems, the data quality can be defined as the "completeness and validity of the data recorded". Data quality can be assessed at a number of levels. The frequency of bias, typos, or "blank" responses on the system can provide a quick and easy indication of data quality. Logical checks can also be performed within the dataset (e.g. material trend curves evaluation and the associated data fitting protocols; anomalies of data distributions, etc).

Full assessments of data quality require special studies to measure the attributes of sensitivity and predictive trend for key parameters of interest. Estimation of the sensitivity of reactor surveillance and safety requires that the data contained in the system is validated and then compared against an external measurement of the true frequency of the material safety related event under surveillance. This external measurement should be a gold standard, such as ASME Code and ASTM Standards. Predictive trend estimation similarly requires the information on data sources reported to the surveillance system to be compared against another standard, which also should be a gold standard, e.g. surveillance records, laboratory reports etc, to calculate the proportion of cases which have correctly recorded each item of information.

In the case of the REACTOR Material Registry a gold standard is not easily available, therefore measurement of sensitivity and predictive trend would require special studies, and is beyond the scope of this data quality assurance protocol. This will be considered as data quality projects in the future.

\subsection{Data Quality Assurance Framework}

A framework for assuring data quality of Reactor Material Database needs to be considered. For the purpose of developing the framework, data quality is defined as "the totality and features of a 
data set that bear on its ability to satisfy the needs that result from the intended use of the data". The data quality attributes of data accuracy ("the extent to which registered data are in conformity with the truth material behavior") and data completeness ("the extent to which all necessary data that could have been registered have actually been registered”) are also defined.

Errors that lead to insufficient data quality in a registry were identified by the database managers or the database users and categorized by the stage in the data collection process at which they can occur, and whether they are attributable to the central coordinating server or to local database sites, as illustrated in Table 5.

Table 5: Causes of error leading to insufficient data quality in a registry

\begin{tabular}{|c|c|}
\hline $\begin{array}{l}\text { Cause of error at } \\
\text { Central coordinating server }\end{array}$ & $\begin{array}{c}\text { Cause of error at } \\
\text { Local databases sites }\end{array}$ \\
\hline \multicolumn{2}{|c|}{ Set up and organization of registry } \\
\hline $\begin{array}{l}\text { Unclear or ambiguous data definitions } \\
\text { Unclear data collection guidelines } \\
\text { Poor data collection form lay-out } \\
\text { Poor interface design } \\
\text { Data overload } \\
\text { Programming errors }\end{array}$ & $\begin{array}{l}\text { Illegible handwriting in data source } \\
\text { Incompleteness of data source } \\
\text { Unsuitable data format in source } \\
\text { Data definition unavailable } \\
\text { Lack of systematic approach } \\
\text { Frequent shift of staff } \\
\text { Programming error }\end{array}$ \\
\hline \multicolumn{2}{|c|}{ Data collection } \\
\hline $\begin{array}{l}\text { No control over adherence to } \\
\text { guidelines/definitions } \\
\text { Insufficient data checks }\end{array}$ & $\begin{array}{l}\text { Non-adherence to guidelines/definitions } \\
\text { Calculation errors } \\
\text { Typing errors } \\
\text { Insufficient data checks at entry } \\
\text { Transcription error }\end{array}$ \\
\hline \multicolumn{2}{|c|}{ Quality Improvement } \\
\hline $\begin{array}{l}\text { Insufficient control over correction of } \\
\text { detected data errors locally } \\
\text { Lack of a clear plan for quality } \\
\text { improvement }\end{array}$ & No correction of detected errors \\
\hline
\end{tabular}

Based on these causes, a framework for assurance of data quality in registries (Table 6) can be developed. It identifies actions to be taken during the set up and organization of the registry to prevent error, actions during data collection to detect errors and actions for quality approval.

Table 6: A framework for data quality assurance in registries. 


\begin{tabular}{|c|c|}
\hline Central coordinating server & Local database sites \\
\hline \multicolumn{2}{|c|}{ Actions taken during set up and organization of registry } \\
\hline At outset of the registry & At outset of participation in registry \\
\hline Compose minimum data set necessary & Assign contact person \\
\hline Establish data definitions & Check reliability and completeness of \\
\hline Establish data collection protocol & extraction sources \\
\hline Define pitfalls in data collection & Standardize correction of data items \\
\hline Compose data checks & Continuously \\
\hline Create user friendly data collection forms & Train data collectors \\
\hline Create quality assurance plan & Motivate data collectors \\
\hline In case of new participating site & Make data definitions available \\
\hline Perform site visit & Keep completed data collection forms \\
\hline Train new participants & Use the registry data for local purposes \\
\hline \multicolumn{2}{|l|}{ Continuously } \\
\hline \multicolumn{2}{|l|}{ Motivate participants } \\
\hline \multicolumn{2}{|l|}{ Communicate with local database sites } \\
\hline In case of changes & In case of changes \\
\hline Adjust system appropriately & Adjust system appropriately \\
\hline Communicate with local sites & Communicate with data collectors \\
\hline \multicolumn{2}{|c|}{ Detection during data collection } \\
\hline During import of data into database & Continuously \\
\hline Perform automatic data checks periodically & Visually inspect completed forms \\
\hline Periodically & Perform automatic data checks \\
\hline Perform local site visits for quality audit & Check completeness of registration \\
\hline \multicolumn{2}{|l|}{ Check collected data variability } \\
\hline \multicolumn{2}{|c|}{ Actions for quality improvement } \\
\hline After data import and data checks & After receiving quality reports \\
\hline \multirow[t]{2}{*}{ Provide local sites with data quality report } & Check detected errors \\
\hline & $\begin{array}{l}\text { Correct inaccurate data and fill in incomplete } \\
\text { data }\end{array}$ \\
\hline Control local correction of data errors & Resolve causes of data errors \\
\hline After data audit or variability check & After receiving feedback \\
\hline Give feedback of results and recommendations & Implement recommended changes \\
\hline Resolve causes of data errors & Communicate with personnel \\
\hline
\end{tabular}

\subsection{Material Database Quality Assurance Protocol}

This framework is used to identify actions needed to be taken at the Material Database Registry to assure data quality. It is also used to scope possible new actions. A final list of actions needs 
to be agreed by registry staff and is the basis to the Material Data Quality Assurance Protocol. The protocol aims to assure the quality of the Reactor Material Registry's data, the database infrastructure, and the registry staff.

The protocol is supported by a number of procedures:

- Data collection protocol

- Data collection form

- Data collection checklist -This checklist is to be attached to each data collection form. It will collect date and signature when the data is collected (research personnel), visually inspected (Registry Leader) and entered (research personnel/surveillance scientist). It will also confirm that a duplicate and validation check has been completed on each case, and any issues arising from this will be commented on.

- Data export form - This form is completed whenever data is exported to users. A signature and date confirms review of line listing and data export. A signature, date and comment confirm any errors which are communicated back by the local databases or users and actions arising.

- Data exclusion form -This form will explain why a case is being excluded from the registry. A file of excluded cases will be kept. This will allow investigation of changes in trends within the data collected by the registry to see if they are due to changes in the data inclusion and/or data exclusion cases.

These documents can be audited to measure performance of the registry. This protocol should be reviewed and updated on an annual basis.

The considerations of preparing the protocol are:

- The scope and purpose of the protocol is clearly defined.

- Relevant institutions are included in its preparation and the target users have been defined.

- The guidelines were developed following a rigorous search of literature on data quality assurance in nuclear material registries, and the recommendations contained link to this literature.

- The protocol is clear and is accompanied by relevant tools for its application in the form of appendices, either in hard copy or electronic file or both, which will facilitate audit.

\subsection{Other Consideration and Recommendation Regarding the QA Protocol}

The user-friendly software package development is an integrated part of database development. It is also an essential part of QA Protocol. A limit effort spent on the consideration of the software development in assisting QA protocol will results in great saving both in time and budget regarding 
QA process. Using a well-designed front end to assembly the QA data in a more presentable format will certainly increase the efficiency of the QA staff and reduce error due to linking QA data for inspection or verification. As regarding "Evaluation" and "Validation" of the QA data, it may certainly require further process, such as transferring the data into a form other than the existing structures to satisfy the specific calibrating formulation or material models.

The success of REACTOR Material Database development will be critically depended on a welldesigned infrastructure between the central server, so call "Common Database," and the local participants, so call "Local Databases." Therefore, a central server build at ORNL utilizing the existing infrastructure is recommended. This central control server provides mechanism for uniform data structure development, data registry and update, data storage, data QA, data archive, and gateway (linking bridge) to communicate with each participant's local database directly for data submitting and retrieval, and provides control access to the end users.

In summary, the advantages of having a well-defined central control mechanism, as illustrated in Figs. 1-3, to Reactor Material Database development are:

$>$ Providing a uniform mechanism regarding the data structure, data collection, QA and long term archive protocol

$>$ Eliminating costly, repetitive reexamination and rework of the databases among the developer (local database sites) each time new issues arises or new organizations must be engaged

$>$ Speed-up information flow and decision making process

\section{ACKNOWLEDGEMENTS}

The authors gratefully acknowledge Dan Naus and Rick Lusk for reviewing this report; and Beverly DiPaolo of Engineer Research and Development Center US Army Corps of Engineers for providing support in relating to DHS Advanced Material Council activity. The research was initially sponsored by NRC RES under DOE contract DE-AC05-00OR22725 with UT-Battelle, LLC. 


\section{REFERENCES}

1. J. A. Wang, S. Ranjit, "PR-EDB: Power Reactor Embrittlement Database Version 3,” ORNL/TM-2006/605, 2008.

2. J. A. Wang, “Embrittlement Data Base, Version 1,” NUREG/CR-6506, ORNL/TM-13327, Nuclear Regulatory Commission, 1998.

3. F. W. Stallmann, J. A. Wang, F. B. K. Kam, B. J. Taylor, "PR-EDB: Power Reactor Embrittlement Data Base, Version 2," NUREG/CR-4816, ORNL/TM-10328/R2, 1994.

4. F. W. Stallmann, J. A. Wang, F. B. K. Kam, "TR-EDB: Test Reactor Embrittlement Data Base, Version 1," NUREG/CR-6076, ORNL/TM-12415, Nuclear Regulatory Commission, January 1994.

5. J. A. Wang, F. B. K. Kam, and F. W. Stallmann, "PT_LIM: A Computer Program to compute Pressure-Temperature Limits for U.S. Power Reactor,” NUREG/CR-6019, ORNL/CAD/TM286, Nuclear Regulatory Commission, 1993.

6. J. A. Wang, F. B. K. Kam, and F. W. Stallmann, "Embrittlement Data Base (EDB) and Its Applications," Effects of Radiation on Materials: 17th Volume, ASTM STP 1270, pp. 500521, August 1996.

7. J. A. Wang, "Development of Embrittlement Prediction Models for U.S. Power Reactors and the Impact of the Heat-Affected Zone to Thermal Annealing," Effects of Radiation on Materials: 18th Volume, ASTM STP 1325, pp. 525-540, March 1999.

8. J. A. Wang, "Analysis of the Irradiated Data for A302B and A533B Correlation Monitor Materials,” Effects of Radiation on Materials: Vol.19, ASTM STP 1366, pp. 59-80, March 2000.

9. J. A. Wang and N. S. Rao, "New Methodologies for Developing Radiation Embrittlement Models and Trend Curves of the Charpy Impact Test Data," Effects of Radiation on Materials, Vol. 21, pp.634-652, ASTM STP 1447, Journal of ASTM International, December, 2004.

10. Jy-An Wang, N.S. Rao, S. Konduri, “The Development of Radiation Embrittlement Models for U. S. Power Reactor Pressure Vessel Steels,” Journal of Nuclear Materials, V. 362 (1), pp. 116-127, 2007.

11. J. A. Wang, F.B.K. Kam, "Review of the International Atomic Energy Agency International Aging Database on Reactor Pressure Vessel Materials and U.S. Nuclear Regulatory Commission/Oak Ridge National Laboratory Embrittlement Data Base,” ORNL/NRC/LTR97/25, February 1998.

12. J. A. Wang, "Comparison of the EDB Program, Charpy Impact Data Fitting to the TANH Program of the EPRI Steel Handbook,” TENERA Project, 1989.

13. I. Remec, J. A. Wang and F. B. K. Kam, "HFIR Steels Embrittlement: The Possible Effects of Gamma Field Contribution,” Effects of Radiation on Materials: 17th Volume, ASTM STP 1270, pp. 591-605, August 1996.

14. Remec, I., Wang, J. A., Kam, F. B. K., and Farrell, K., "Effects of Gamma-Induced Displacements on HFIR Pressure Vessel Materials," Journal of Nuclear Material, Volume 217 pp. 258-268, 1994. 